\title{
Soil and Plant Analyses to Diagnose Hop Fields Irregular Growth
}

\author{
Sandra Afonso ${ }^{1}$ (D) - Margarida Arrobas ${ }^{1}$ (D) $\cdot$ M. Ângelo Rodrigues ${ }^{1}$ (i)
}

Received: 28 February 2020 / Accepted: 21 May 2020

(C) Sociedad Chilena de la Ciencia del Suelo 2020

\begin{abstract}
In cultivated fields, patches of poorly developed vegetation often appear without a clear indication of what is affecting the growth of the plants. The purpose of this study was to investigate the causes behind these irregularities in the hop (Humulus lupulus L.) fields of NE Portugal which are greatly reducing crop yield and farmers' profits and to provide guidance to farmers as to appropriate remedial action. Patches of different levels of plant development were selected within hop fields and categorized according to plant vigour (weak, fair and good). Several soil properties were determined and related to the plant nutritional status and dry matter yield of different parts of the plant (hops, leaves, stems). Data was subjected to analysis of variance and principal component analysis. The results suggest that crop yield is reduced mostly due to poor soil aeration and excessive soil and tissue $\mathrm{Mn}$ and Fe levels. The plants from the plots of weaker vigour seem to be particularly affected by toxic levels of Mn and the plants from the plots of fair vigour by Fe levels. $\mathrm{pH}$, texture (clay content), cation exchange capacity and organic carbon seem to be other soil properties with some degree of influence on plant performance. From these results, farmers are advised to increase soil aeration by implementing a drainage system and converting to a drip irrigation system, in addition to increasing soil $\mathrm{pH}$ by liming to reduce Mn toxicity.
\end{abstract}

Keywords Humulus lupulus $\cdot$ Soil properties $\cdot$ Nutritional disorders $\cdot$ Waterlogging $\cdot$ Plant growth and yield

\section{Introduction}

Hop (Humulus lupulus L.) is a perennial species that has a productive life of more than 20 years. In the north-eastern of Portugal, cultivated hop fields sometimes display patches of heterogeneous plant vigour, even when managed by the same farmer and subjected to similar cropping techniques. This is an important issue since the loss of production is progressively reducing the farmers' profit. The causes are often not easily identified, but they may be related to soil properties and plant nutritional disorders.

Soil compaction and soil water content are spatially and temporarily variable factors which greatly influence soil

M. Ângelo Rodrigues

angelor@ipb.pt

Sandra Afonso

sandraafonso@ipb.pt

Margarida Arrobas

marrobas@ipb.pt

1 Centro de Investigação de Montanha, Instituto Politécnico de Bragança, Campus de Santa Apolónia, 5300-253 Bragança, Portugal available nutrients (Weil and Brady 2017). In Portugal, hop farmers usually irrigate the crop by flooding the space between rows (Rodrigues et al. 2016). This system negatively impacts the soils, causing the appearance of areas with excessive or insufficient water content and increasing soil compaction, which demands frequent tillage, with consequences for soil properties, plant nutritional status and plant health (Rodrigues and Morais 2015).

In Mediterranean climates, most of the rain falls during the winter months (mainly between December and March), which in poorly drained soils, may be a serious problem for winter and perennial crops. Flooding, caused by rain and summer surface irrigation, greatly influences the soil redox potential and consequently its chemical and physical properties (Favre et al. 2002). The first measurable effect induced by flooding is the disappearance of oxygen $\left(\mathrm{O}_{2}\right)$ (Parent et al. 2008; Irfan et al. 2010). Plant roots need a regular $\mathrm{O}_{2}$ supply for mitochondrial respiration. The availability of $\mathrm{O}_{2}$ also regulates oxidation-reduction reactions, with a major influence on the bioavailability of several essential nutrients (Weil and Brady 2017). Reduced levels of $\mathrm{O}_{2}$ in the soil favour the activity of anaerobic microorganisms which increases denitrification, with the emission of important nitrogen gases, such as dinitrogen $\left(\mathrm{N}_{2}\right)$ and/or nitrous oxides $\left(\mathrm{NO}_{x}\right)$, into the 
atmosphere (Xia et al. 2019). Furthermore, flooding stress may reduce plant dry matter yield (Gao et al. 2020).

During the thermodynamic sequence of the reduction process, nitrate reduction is followed by the reduction of $\mathrm{Mn}, \mathrm{Fe}$ and sulphate (Osman 2013). The decrease in redox potential is followed by the increase in $\mathrm{pH}$ and soluble $\mathrm{Fe}, \mathrm{Mn}, \mathrm{NH}_{4}{ }^{+}, \mathrm{Ca}$, $\mathrm{Mg}, \mathrm{K}$ and $\mathrm{Na}$ (Phillips and Greenway 1998; Pezeshki and DeLaune 2012). Toxic products, such as ethanol, lactic acid, acetaldehyde, aliphatic acids and cyanogenic compounds, are also produced under reduction conditions (Parent et al. 2008; Pezeshki and DeLaune 2012). The accumulation of these compounds along with the increased available forms of $\mathrm{Fe}$ and $\mathrm{Mn}$ can have deleterious effects on plants (Millaleo et al. 2010, 2019; Pezeshki and DeLaune 2012). The effects of flooding on plants can be diverse and may include deficiency of some and/or toxicity of other nutrients (Stieger and Feller 1994; Pezeshki and DeLaune 2012) and reduction in photosynthesis and biomass accumulation (George et al. 2012; Sparrow and Uren 2014).

Organic matter is a key component of soil fertility and an important factor in soil structure formation (Osman 2013). In general, organic matter favourably influences the physical, biological and chemical properties of the soil. Among many other favourable effects on soil, organic matter reduces compaction, favours aeration and water holding capacity, enhances nutrient cycling and releases valuable nutrients for root uptake (Tan 2011; Singer and Munns 2002). The organic matter content of a soil depends on the annual input of organic debris (crop residues or manure), soil properties (such as texture), climate (precipitation and temperature) and agricultural practices, such as the intensity of soil tillage (Weil and Brady 2017).

Soil $\mathrm{pH}$ is another major factor affecting nutrient availability and plant growth (Bindraban et al. 2015). Depending on soil $\mathrm{pH}$, several essential nutrients become more or less available for root uptake. The bioavailability of the vast majority of essential nutrients increases with $\mathrm{pH}$ close to neutrality. As $\mathrm{pH}$ moves away from neutrality, the risk of deficiency or toxicity of certain elements increases. In alkaline soils, for instance, there is a high risk of Fe deficiency, while in acidic soils, the risk of Al and Mn toxicity increases (George et al. 2012). Plant species differ in their preferences for soil $\mathrm{pH}$. Some species thrive in acidic soils while others prefer alkaline soils. Most, however, thrive best in near-neutral or slightly acidic soils (Tan 2011). The $\mathrm{pH}$ range considered to be the most adequate for hop is between 6.0 and 6.7 (Gingrich et al. 1994; Čeh and Čremožnik 2015).

The availability of essential nutrients, both macronutrients and micronutrients, is of utmost importance for plant performance. Soil nutrient availability depends on natural factors such as soil texture and mineralogy. Clay soils, for example, may fix and/or adsorb high quantities of some nutrients that are progressively released to plants (Osman 2013). Some agricultural practices, such as management of crop residues and manure application, also have long-term effects on soil nutrient availability (Lipecki and Berbeć 1997; Samson et al. 2019).

The main goal of this study is to try to establish the causes for the appearance of heterogeneous areas of poorly developed plants in commercial hop fields in NE of Portugal. The hypothesis set for this work is that anoxia, and its multiple relationships with other soil properties, and the availability of plant nutrients are responsible for the poor vigour of plants. Thus, the objectives of the study are (i) to relate several soil properties, such as soil $\mathrm{pH}$, texture, organic carbon, electrical conductivity, exchangeable bases and extractable macronutrients and micronutrients, to elemental plant composition (leaves, stems and flowers); (ii) to relate all those soil and plant variables to aboveground dry matter yield and, in particular, to hop cone dry matter yield; and (iii) to seek a diagnosis that will guide farmers to intervene in the fields to make them more uniform, eliminating the patches of low-vigour plants which are responsible for reduced dry matter yield. To this aim, these were selected for the study plots previously classified by farmers as producing plants of weak, fair or good vigour.

\section{Materials and Methods}

\subsection{Characterization of Field Plots and Sample Collection}

The hop fields used for this study are located in Bragança, NE of Portugal, a region that benefits from a Mediterranean-type climate (average annual air temperature of $12.7^{\circ} \mathrm{C}$; annual precipitation of $772.8 \mathrm{~mm}$ ) (IPMA 2020). The soil and plant samples were collected during the growing season of 2016, in two hop farms located in Pinela $\left(41^{\circ} 40^{\prime} 33.6^{\prime \prime} \mathrm{N}, 6^{\circ} 44^{\prime} 32.7^{\prime \prime}\right.$ W) and Vinhas ( $\left.41^{\circ} 33^{\prime} 34.8^{\prime \prime} \mathrm{N}, 6^{\circ} 48^{\prime} 42.9^{\prime \prime} \mathrm{W}\right)$. Each farmer grows approximately a total of 6 ha of hop of the cultivar Nugget. The fields are grown in a 7-m conventional hightrellis system, with concrete poles, connected with cable, in a ' $\mathrm{V}$ ' design system. In Vinhas hop farm, there are several plots showing a markedly different vegetative pattern in terms of the vigour of hop plants, previously classified by the farmers as weak, fair and good. In Pinela farm, hop plants generally present a fair or good vigour. Soil and plant samples were separately collected in the different vigour plots. Seven plots were selected corresponding to weak (plot 1, plot 2, plot 3), fair (plot 4, plot 5) and good (plot 6, plot 7) plant vigour. Plots 1, 2, 3 and 4 were marked in Vinhas farm and plots 5, 6 and 7 in Pinela farm.

Both farmers manage their fields by a surface irrigation system consisting of flooding the space between rows. Several tillage passes ( 3 to 4 ) are performed every year to remove the crusts and allowing water infiltration. Both 
farmers used to apply a compound NPK (7:14:14) fertilizer early in the spring, followed by two applications of $\mathrm{N}$ as sidedress, totalling 150, 44 (100) and 83 (100) $\mathrm{kg} \mathrm{N}, \mathrm{P}$ $\left(\mathrm{P}_{2} \mathrm{O}_{5}\right)$ and $\mathrm{K}\left(\mathrm{K}_{2} \mathrm{O}\right) \mathrm{ha}^{-1}$. The farmer of Pinela also uses to apply $40 \mathrm{t} \mathrm{ha}^{-1}$ of farmyard manure. Both farmers follow a phytosanitary program to protect the crop from pests and diseases.

Composite soil samples (15 sampling points per composite sample) were randomly collected at $0-20 \mathrm{~cm}$ depth, between rows, on June 2, with three replicates per plot. The soil of a composite sample was homogenized in a bucket and separated from the larger stones. A fraction of $\sim 500 \mathrm{~g}$ soil was placed in a labelled bag and sent to the laboratory. Three samples of 30 leaves (blades plus petioles) were collected per plot in three dates evenly spaced (June 2, July 1 and July 27) during the growing season. In the first sampling date, the leaves were collected at $\sim 1 \mathrm{~m}$ in height. In the second and third sampling dates, leaves were collected at $\sim 1 \mathrm{~m}$ and $\sim 2 \mathrm{~m}$ in height. At the harvest (September 1), the aboveground biomass of six random plants per plot was separated in two different leaf samples (basal leaves [to $2 \mathrm{~m}$ in height] and top leaves [last $2 \mathrm{~m}$ from the top]), stems and hop cones. By analysing several times, different tissues and tissues at different positions in the canopy, a more complete diagnosis of the nutritional status of the plants is potentially obtained. The samples of the aboveground biomass taken at harvest were weighed in fresh and separated into stems, leaves and cones. Subsamples of each plant part were weighed again and subsequently oven-dried at $65^{\circ} \mathrm{C}$ and weighed dry for the estimation of the dry matter yield of the original field samples. Thereafter, three subsamples of each plant tissue were ground $\left(1-\mathrm{mm}^{2}\right.$ sieve $)$ and analysed for elemental composition.

\subsection{Laboratory Analysis}

Soil samples were oven-dried at $40{ }^{\circ} \mathrm{C}$ and sieved in a mesh of $2 \mathrm{~mm}$. Samples were analysed for texture (pipette method), $\mathrm{pH}$ $\left(\mathrm{H}_{2} \mathrm{O}\right.$ and $\left.\mathrm{KCl} 1 \mathrm{M}\right)$, electrical conductivity of soil extract (soil:solution, 1:2.5), exchangeable complex (ammonium acetate, $\mathrm{pH}$ 7.0) and organic carbon (Walkley-Black method). The readily soluble forms of $\mathrm{P}$ and $\mathrm{K}$ were extracted by a combination of ammonium lactate and acetic acid buffered at $\mathrm{pH} 3.7$ (Egner-Riehm method). Extractable P was also determined by the Olsen method. Phosphate in the extract was determined calorimetrically with the blue ammonium molybdate method using ascorbic acid as reducing agent. Soil B was extracted by hot water and the extracts analysed by the azomethine $\mathrm{H}$ method. For more details of these analytical procedures, the reader is referred to Van Reeuwijk (2002). The availability of other micronutrients in soil $(\mathrm{Cu}, \mathrm{Fe}, \mathrm{Zn}$ and $\mathrm{Mn})$ was determined by atomic absorption spectrometry after extraction with ammonium acetate and EDTA, according to the method described by Lakanen and Erviö (1971). Soil acid phosphatase activity was determined as proposed by Tabatabai and Bremner (1969) and Eivazi and Tabatabai (1977).

Elemental analyses of leaf, stem and cone samples were performed by Kjeldahl $(\mathrm{N})$, colorimetry (B and $\mathrm{P}$ ), flame emission spectrometry $(\mathrm{K})$ and atomic absorption spectrophotometry $(\mathrm{Ca}, \mathrm{Mg}, \mathrm{Cu}, \mathrm{Fe}, \mathrm{Zn}$ and $\mathrm{Mn})$ methods after nitric digestion of the samples (Walinga et al. 1989). Additionally, nitrate concentration in hop cones was determined by UV-vis spectrophotometry in a water extract (dry tissue:solution, 2.5:50) (Clescerl et al. 1998).

\subsection{Data Analysis}

Data from soil and plant samples was subjected to one-way (plots of different plant vigour) analysis of variance (ANOVA), by using IBM SPSS v.25.0 program. When significant differences occurred, the means were separated by the Tukey-Kramer HSD test $(\alpha=0.05)$. A correlation analysis was performed for soil data with the Pearson coefficient when the assumption of normality and linearity was accomplished, and not being the case, with the Spearman coefficient. The principal component analysis (PCA) was also applied to soil and plant data by a symmetrical method. The components were retained, considering the eigenvalues superior to 1 and the scree plot. Internal consistency was measured with Cronbach's alpha. Subsequently, the differences between plots in the components extracted were subjected to ANOVA and Tukey-Kramer HSD test $(\alpha=0.05)$.

\section{Results}

\subsection{Dry Matter}

Plants classified as having higher vigour (good) in the function of their development in the previous years produced higher average total dry matter (Fig.1). Values of plot 6 were significantly higher than those of plot 5 and plots of weaker plants (1, 2 and 3). Total dry mater yield reached the highest average values in plot $6\left(2587 \mathrm{~g} \mathrm{plant}^{-1}\right)$ and the lowest average value in plot $1\left(513 \mathrm{~g} \mathrm{plant}^{-1}\right)$. The difference in cone production was even higher when comparing the plots of the plants of good vigour with those of fair and weak vigour. The highest $\left(1193 \mathrm{~g} \mathrm{plant}^{-1}\right)$ and the lowest $\left(75 \mathrm{~g} \mathrm{plant}^{-1}\right)$ values were found in plots 6 and 1, respectively. In turn, the differences in leaf and stem production were less marked between fair and good plots, though a significant difference had persisted.

\subsection{Soil Properties}

Soil texture in plots 1, 2 and 3 (weak plant vigour) varied between sandy clay loam, loam and silty clay loam (Fig. 2). 
Fig. 1 Average dry matter yield $(n=6)$ separated by plant parts (leaves, stems and cones) in the seven plots of plants with different vigour (weak, fair and good). For each plant part, means followed by different letters are significantly different by the Tukey HSD test $(\alpha=0.05)$

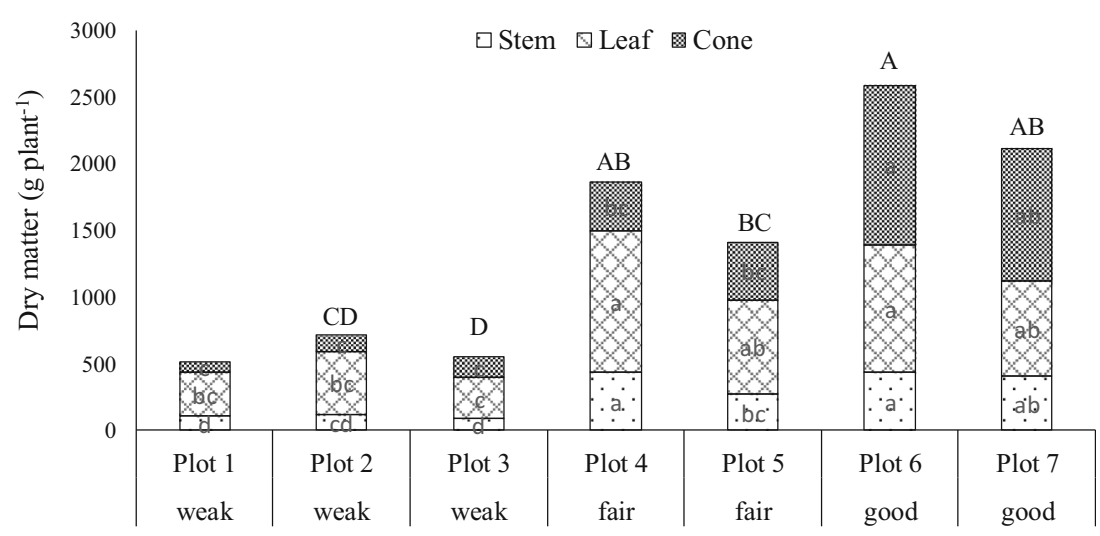

The soils of the plots with fair plant vigour (plots 4 and 5) were clay loam. The soil of plots 6 and 7 (good plant vigour) varied between sandy clay loam and loam.

Soil $\mathrm{pH}\left(\mathrm{H}_{2} \mathrm{O}\right)$ significantly varied among experimental plots (Table 1). The lower (5.1) and higher (6.2) values were found in plots 7 and 2, respectively. Conductivity and acid phosphatase activity also significantly varied among plots. Conductivity ranged from $51.7 \mu \mathrm{s} \mathrm{m} \mathrm{m}^{-1}$ in plot 6 to $99 \mu \mathrm{s} \mathrm{m}^{-1}$ in plot 3 . The higher values of acid phosphatase activity occurred in plots 5, 4 and 3 which correspond to plants of fair (plots 4 and 5) and weak (plot 3) vigour. Organic carbon, for instance, did not significantly vary among experimental plots. Average values varied from $7.3 \mathrm{~g} \mathrm{~kg}^{-1}$ (plot 6) to $16.7 \mathrm{~g} \mathrm{~kg}^{-1}$ (plot 4). Extractable P significantly varied among experimental plots as determined by both the analytical methods. The higher average values were found in plot 5 and the lower values in plot 3. Extractable $\mathrm{K}$ varied from $286 \mathrm{mg} \mathrm{kg}^{-1}$ (plot 7) to $93.0 \mathrm{mg} \mathrm{kg}^{-1}$ (plot 3), the differences being statistically significant. Exchangeable bases (Ca, Mg, K and $\mathrm{Na}$ ) and exchangeable acidity significantly varied among experimental plots. The higher average values of exchangeable bases were found in plots 4 and 5. Exchangeable acidity was higher in plot 7 and lower in plots 1, 4 and 6. The average values of Fe, $\mathrm{Mn}, \mathrm{Zn}, \mathrm{Cu}$ and $\mathrm{B}$ were higher in plots 2, 1, 2, 3 and 5, respectively. The values of $\mathrm{Fe}, \mathrm{Mn}, \mathrm{Zn}$ and $\mathrm{Cu}$ were lower in plot 7, and B values were lower in plot 3 .
The results for significant correlation coefficients between soil properties are shown in Table 2 . No significant correlations were found for extractable $\mathrm{P}, \mathrm{K}$ and $\mathrm{B}$ and exchangeable $\mathrm{K}$ and $\mathrm{Na}$; hence, results are not shown. Correlations were significant and positive between $\mathrm{pH}\left(\mathrm{H}_{2} \mathrm{O}\right.$ and $\left.\mathrm{KCl}\right)$ and organic $\mathrm{C}, \mathrm{CEC}$, exchangeable $\mathrm{Ca}$ and $\mathrm{Mg}$ and acid phosphatase activity, indicating that these variables increase to higher $\mathrm{pH}$ values. Significant and positive correlations were found between $\mathrm{pH}$ and the micronutrients $\mathrm{Fe}, \mathrm{Cu}$ and $\mathrm{Zn}$. For $\mathrm{Mn}$, a moderated and negative correlation with conductivity was found, suggesting that when conductivity is high, Mn is moderately low. Exchangeable acidity presented a moderated positive correlation with conductivity. Texture, CEC, exchangeable $\mathrm{Ca}$, exchangeable $\mathrm{Mg}$ and acid phosphatase activity were strong and positively correlated to clay and negatively correlated to sand.

\subsection{Nutrient Concentration in Plant Tissues}

In the first sampling date, leaf $\mathrm{N}$ concentrations were higher in the plots corresponding to plants of higher vigour (Table 3). The average values varied from $40.0 \mathrm{~g} \mathrm{~kg}^{-1}$ (plot 1, weak) to $46.1 \mathrm{~g} \mathrm{~kg}^{-1}$ (plot 7, good). In the next sampling dates, there was an observed and opposite trend, in particular in the lower strata of the plants, probably due to a dilution effect by the increase in plant biomass in the most productive plots. Plot 7 sometimes
Fig. 2 Soil texture of the samples $(n=3)$ collected between rows at a $0-20 \mathrm{~cm}$ depth in hop plots with plants of weak, fair and good vigour

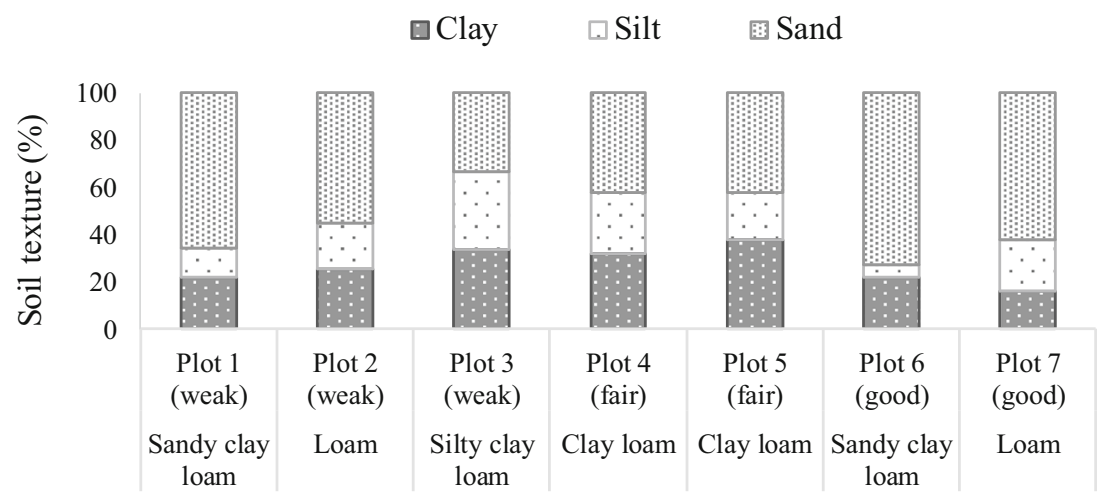




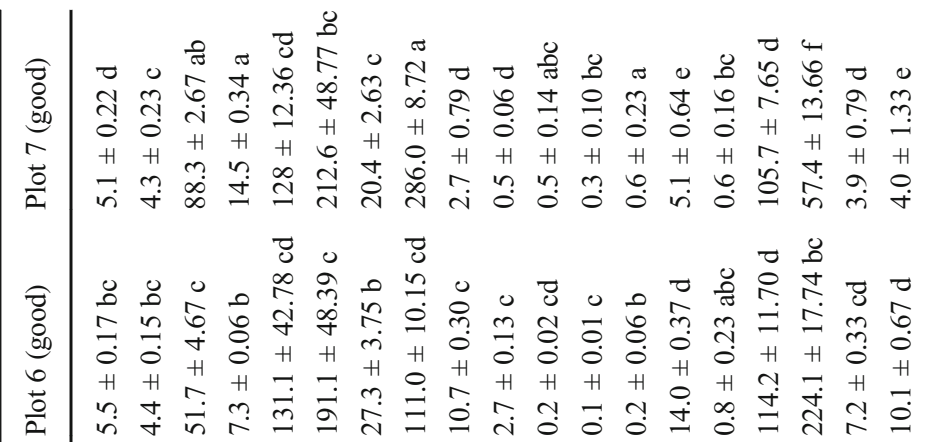

ช

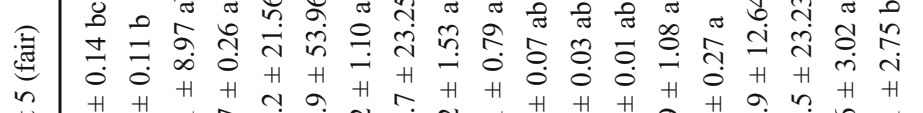

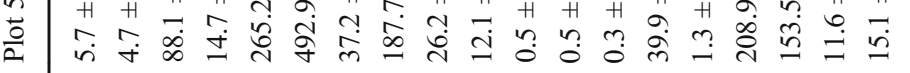

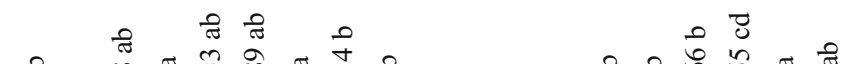

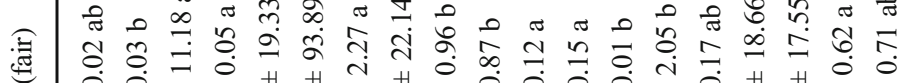

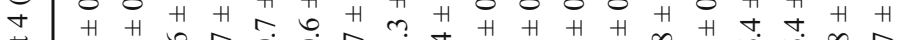

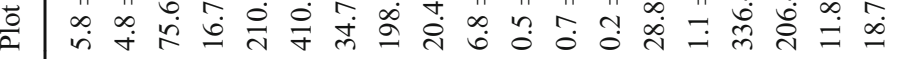

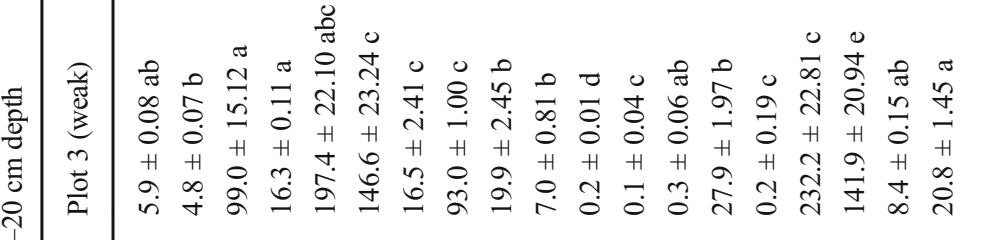

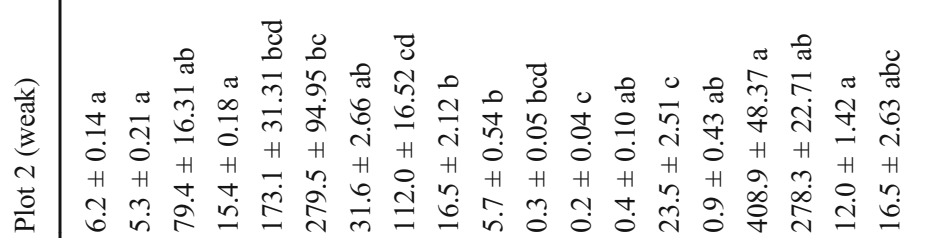

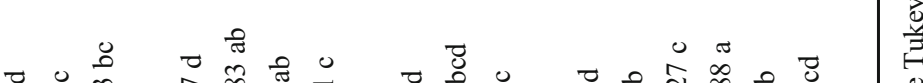

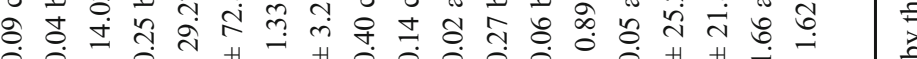

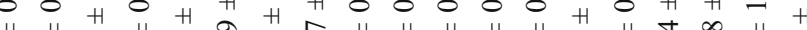

H H

䒯

on

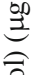

窇

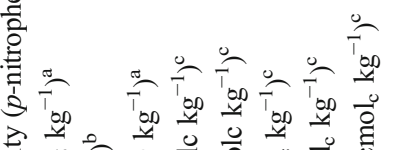

T⿸广土

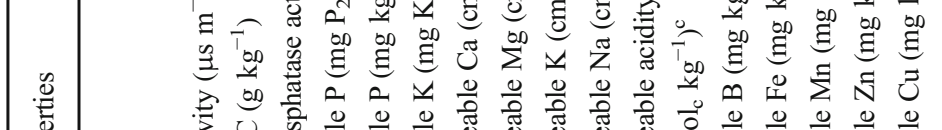


Table 2 Significant correlation coefficients of conductivity, $\mathrm{pH}\left(\mathrm{H}_{2} \mathrm{O}\right.$ and $\left.\mathrm{KCl}\right)$ and texture to soil properties

\begin{tabular}{|c|c|c|c|c|c|c|}
\hline & Conduct. & $\mathrm{pH}_{\mathrm{H} 2 \mathrm{O}}$ & $\mathrm{pH}_{\mathrm{KCl}}$ & Clay & Silt & Sand \\
\hline Organic C & $0.75 * * \dagger$ & $0.49^{* *}$ & $0.58 * * *$ & $0.52 * *$ & $0.87 * * \dagger$ & $-0.84 * *+$ \\
\hline Cation exchange capacity & & $0.61 * * *$ & $0.63 * * \$$ & $0.95 * * \hbar$ & $0.44^{\dagger \dagger}$ & $-0.71 * *$ \\
\hline Exchangeable $\mathrm{Ca}$ & & $0.61 * * *$ & $0.61 * * *$ & $0.95 * * *$ & & $-0.71 * * \ddagger$ \\
\hline Exchangeable Mg & & $0.61 * * *$ & $0.61 * * *$ & $0.95 * * *$ & & $-0.70 * * \ddagger$ \\
\hline Exchangeable acidity & $0.47 * *$ & & & & & \\
\hline Acid phosphatase activity & $0.50^{* \dagger}$ & $0.56^{* *}$ & $0.59 * *$ & $0.83 * * *$ & $0.50^{* \pm}$ & $-0.72 * * *$ \\
\hline Conductivity & & & & & $0.63 * \ddagger$ & $-0.61 * * \dot{t}$ \\
\hline $\mathrm{Fe}$ & & $0.77 * * \dagger$ & $0.81 * * \dagger$ & & & $-0.46^{* \hbar}$ \\
\hline $\mathrm{Mn}$ & $-0.51 * \hbar$ & & & & $-0.63 * * 末$ & \\
\hline $\mathrm{Zn}$ & & $0.68 * * \dagger$ & $0.67 * * \dagger$ & $0.62 * * *$ & & $-0.46^{* \star}$ \\
\hline $\mathrm{Cu}$ & & $0.77 * * *^{\dagger}$ & $0.66^{* * \dagger}$ & $0.76^{* * *}$ & $0.54^{\ddagger \ddagger}$ & $-0.80 * * \hbar$ \\
\hline
\end{tabular}

*Significant at 0.05 level

**Significant at 0.01 level

${ }^{\dagger}$ Pearson coefficient

\$ Spearman coefficient

appeared as an exception with values similar to those of plot 1 . At harvest, the plots of plants of lower vigour also tended to present higher leaf $\mathrm{N}$ concentration than those of higher vigour (see plot 6) and the same is true for stems and cones.

Leaf $P$ concentrations varied from the first to the last sampling date (Table 4). The highest average value $\left(3.5 \mathrm{~g} \mathrm{~kg}^{-1}\right)$ was found in plot 1 in the sampling on June 2 and the lowest average value $\left(1.0 \mathrm{~g} \mathrm{~kg}^{-1}\right)$ in plot 6 on September 1 . In spite of significant differences among plots had been found for some sampling dates, there was not observed a coherent trend related to plant vigour. Cone and stem $\mathrm{P}$ concentrations also varied among plots, but a relation with plant vigour was also not observed.

Tissue K concentrations significantly varied in all sampling dates. Plants with good vigour (plot 7) clearly exhibit the higher values in all tissues, except in stems where weak plants in plot 1 presented the higher $\mathrm{K}$ concentration (Table 5). The lower $\mathrm{K}$ values were recorded for plants with weak vigour in plot 3. Leaf $\mathrm{K}$ concentrations ranged from $3.6 \mathrm{~g} \mathrm{~kg}^{-1}$ for weak plants in plot 3 at harvest to $29.2 \mathrm{~g} \mathrm{~kg}^{-1}$ for good vigour plants in plot 7 on July 1 and for leaves taken at $2 \mathrm{~m}$. In the cones, the higher and lower average leaf $\mathrm{K}$ values were $18.9 \mathrm{~g} \mathrm{~kg}^{-1}$ and $10.7 \mathrm{~g} \mathrm{~kg}^{-1}$, which were found in plots 7 and 3, respectively.

For the other macronutrients analysed ( $\mathrm{Ca}$ and $\mathrm{Mg}$ ), full tables were not provided to reduce the length of the texts. However, leaf Ca levels significantly increased throughout the growing season. On June 2, the values varied from $4.6 \mathrm{~g} \mathrm{~kg}^{-1}$ (plot 7) to $10.5 \mathrm{~g} \mathrm{~kg}^{-1}$ (plot 4) and on September 1 from $15.5 \mathrm{~g} \mathrm{~kg}^{-1}$ (plot 5) to $49.2 \mathrm{~g} \mathrm{~kg}^{-1}$ (plot 1). Leaf Ca concentrations significantly varied among experimental plots for all sampling dates. However, a coherent trend regarding plots or plant vigour was not found. Stems and cones presented $\mathrm{Ca}$ concentrations lower than the leaves collected at harvest. In spite of there had been found significant differences among plots, a coherent trend regarding plant vigour was also not observed.

Leaf $\mathrm{Mg}$ concentrations followed the trend observed for $\mathrm{Ca}$. The results showed an increase of leaf $\mathrm{Mg}$ over the

Table 3 Tissue nitrogen concentrations $\left(\mathrm{g} \mathrm{kg}^{-1}\right)$ (mean \pm standard deviation) for different sampling dates and tissue position in the plant from the different experimental plots

\begin{tabular}{|c|c|c|c|c|c|c|c|c|}
\hline Date & $\begin{array}{l}\text { Tissue } \\
\text { (plant position) }\end{array}$ & Plot 1 (weak) & Plot 2 (weak) & Plot 3 (weak) & Plot 4 (fair) & Plot 5 (fair) & Plot 6 (good) & Plot 7 (good) \\
\hline June 2 & Leaf $(1 \mathrm{~m})$ & $40.0 \pm 1.90 \mathrm{~b}$ & $40.2 \pm 1.12 b$ & $45.8 \pm 0.86 \mathrm{a}$ & $45.0 \pm 2.20 \mathrm{a}$ & $43.3 \pm 1.63 \mathrm{ab}$ & $45.3 \pm 2.46 \mathrm{a}$ & $46.1 \pm 0.44 \mathrm{a}$ \\
\hline \multirow[t]{2}{*}{ July 1} & Leaf (1 m) & $39.5 \pm 0.92 \mathrm{ab}$ & $38.4 \pm 2.01 \mathrm{ab}$ & $35.9 \pm 1.50 \mathrm{bc}$ & $33.8 \pm 1.27 \mathrm{~cd}$ & $36.0 \pm 0.44 \mathrm{bc}$ & $32.0 \pm 2.40 \mathrm{~d}$ & $40.1 \pm 1.26 \mathrm{a}$ \\
\hline & Leaf $(2 \mathrm{~m})$ & $40.6 \pm 1.17 \mathrm{a}$ & $42.5 \pm 0.81 \mathrm{a}$ & $38.2 \pm 2.81 \mathrm{ab}$ & $32.3 \pm 1.22 \mathrm{c}$ & $34.7 \pm 1.36 \mathrm{bc}$ & $30.4 \pm 2.95 \mathrm{c}$ & $39.5 \pm 1.56 \mathrm{a}$ \\
\hline \multirow[t]{2}{*}{ July 27} & Leaf $(1 \mathrm{~m})$ & $38.1 \pm 1.88 \mathrm{a}$ & $36.5 \pm 0.75 \mathrm{ab}$ & $32.6 \pm 0.55 \mathrm{c}$ & $30.9 \pm 2.55 \mathrm{c}$ & $33.8 \pm 0.10 \mathrm{bc}$ & $33.2 \pm 1.25 \mathrm{bc}$ & $36.2 \pm 1.05 \mathrm{ab}$ \\
\hline & Leaf (2 m) & $44.7 \pm 0.69 \mathrm{a}$ & $42.0 \pm 0.44 \mathrm{~b}$ & $36.1 \pm 1.48 \mathrm{c}$ & $38.3 \pm 0.40 \mathrm{c}$ & $37.7 \pm 1.36 \mathrm{c}$ & $37.7 \pm 0.85 \mathrm{c}$ & $38.6 \pm 1.25 \mathrm{c}$ \\
\hline \multirow[t]{4}{*}{ September 1 (harvest) } & Leaf (basal) & $32.0 \pm 1.90 \mathrm{a}$ & $29.1 \pm 1.16 \mathrm{abc}$ & $26.5 \pm 1.05 \mathrm{bc}$ & $25.8 \pm 3.52 \mathrm{c}$ & $27.0 \pm 2.83 \mathrm{abc}$ & $24.4 \pm 1.21 \mathrm{c}$ & $31.5 \pm 1.03 \mathrm{ab}$ \\
\hline & Leaf (top) & $35.8 \pm 1.29 \mathrm{a}$ & $32.6 \pm 0.57 \mathrm{ab}$ & $29.8 \pm 0.45 \mathrm{~b}$ & $32.0 \pm 2.10 \mathrm{ab}$ & $32.4 \pm 2.08 \mathrm{ab}$ & $30.4 \pm 1.61 \mathrm{~b}$ & $35.0 \pm 2.06 \mathrm{a}$ \\
\hline & Stem (total) & $12.5 \pm 0.15 \mathrm{a}$ & $10.8 \pm 0.61 b$ & $10.4 \pm 1.11 \mathrm{~b}$ & $9.3 \pm 0.70 \mathrm{bc}$ & $8.6 \pm 0.92 \mathrm{c}$ & $8.3 \pm 0.29 \mathrm{c}$ & $8.1 \pm 0.15 \mathrm{c}$ \\
\hline & Cone (total) & $31.9 \pm 2.79 \mathrm{a}$ & $29.0 \pm 0.76 \mathrm{a}$ & $29.4 \pm 3.25 \mathrm{a}$ & $31.8 \pm 2.00 \mathrm{a}$ & $29.5 \pm 1.00 \mathrm{a}$ & $29.3 \pm 2.14 \mathrm{a}$ & $22.5 \pm 0.76 b$ \\
\hline
\end{tabular}

Means followed by different letters in rows are significantly different by the Tukey HSD test $(\alpha=0.05)$ 
Table 4 Tissue phosphorus concentrations $\left(\mathrm{g} \mathrm{kg}^{-1}\right)$ (mean \pm standard deviation) at different sampling dates and tissue position in the plant from the different experimental plots

\begin{tabular}{lllllllll}
\hline Date & $\begin{array}{l}\text { Tissue (plant } \\
\text { position) }\end{array}$ & Plot 1 (weak) & Plot 2 (weak) & Plot 3 (weak) & Plot 4 (fair) & Plot 5 (fair) & Plot 6 (good) & Plot 7 (good) \\
& & & & & & \\
\hline June 2 & Leaf $(1 \mathrm{~m})$ & $3.5 \pm 0.0 \mathrm{a}$ & $2.9 \pm 0.26 \mathrm{~b}$ & $3.0 \pm 0.06 \mathrm{~b}$ & $2.9 \pm 0.20 \mathrm{~b}$ & $3.0 \pm 0.12 \mathrm{~b}$ & $3.1 \pm 0.17 \mathrm{~b}$ & $3.1 \pm 0.06 \mathrm{~b}$ \\
July 1 & Leaf $(1 \mathrm{~m})$ & $2.0 \pm 0.20 \mathrm{ab}$ & $1.9 \pm 0.21 \mathrm{~b}$ & $1.9 \pm 0.20 \mathrm{~b}$ & $2.2 \pm 0.06 \mathrm{ab}$ & $2.0 \pm 0.06 \mathrm{ab}$ & $2.0 \pm 0.21 \mathrm{ab}$ & $2.4 \pm 0.15 \mathrm{a}$ \\
& Leaf $(2 \mathrm{~m})$ & $2.5 \pm 0.06 \mathrm{a}$ & $2.6 \pm 0.10 \mathrm{a}$ & $2.8 \pm 0.25 \mathrm{a}$ & $2.8 \pm 0.12 \mathrm{a}$ & $2.8 \pm 0.15 \mathrm{a}$ & $2.6 \pm 0.25 \mathrm{a}$ & $2.6 \pm 0.12 \mathrm{a}$ \\
July 27 & Leaf $(1 \mathrm{~m})$ & $1.4 \pm 0.06 \mathrm{a}$ & $1.4 \pm 0.06 \mathrm{a}$ & $1.6 \pm 0.06 \mathrm{a}$ & $1.5 \pm 0.20 \mathrm{a}$ & $1.5 \pm 0.06 \mathrm{a}$ & $1.4 \pm 0.20 \mathrm{a}$ & $1.4 \pm 0.06 \mathrm{a}$ \\
& Leaf $(2 \mathrm{~m})$ & $1.6 \pm 0.15 \mathrm{a}$ & $1.8 \pm 0.06 \mathrm{a}$ & $1.9 \pm 0.06 \mathrm{a}$ & $1.7 \pm 0.15 \mathrm{a}$ & $1.7 \pm 0.06 \mathrm{a}$ & $1.9 \pm 0.36 \mathrm{a}$ & $1.5 \pm 0.10 \mathrm{a}$ \\
September 1 & Leaf (basal) & $1.2 \pm 0.12 \mathrm{abc}$ & $1.1 \pm 0.10 \mathrm{bc}$ & $1.4 \pm 0.01 \mathrm{abc}$ & $1.5 \pm 0.36 \mathrm{a}$ & $1.5 \pm 0.06 \mathrm{ab}$ & $1.0 \pm 0.01 \mathrm{c}$ & $1.4 \pm 0.012 \mathrm{abc}$ \\
(harvest) & Leaf (top) & $1.5 \pm 0.20 \mathrm{a}$ & $1.5 \pm 0.06 \mathrm{a}$ & $1.6 \pm 0.06 \mathrm{a}$ & $1.9 \pm 0.40 \mathrm{a}$ & $1.7 \pm 0.35 \mathrm{a}$ & $1.4 \pm 0.12 \mathrm{a}$ & $1.5 \pm 0.10 \mathrm{a}$ \\
& Stem (total) & $1.2 \pm 0.15 \mathrm{~b}$ & $1.0 \pm 0.12 \mathrm{bc}$ & $1.1 \pm 0.15 \mathrm{bc}$ & $1.7 \pm 0.32 \mathrm{a}$ & $1.7 \pm 0.17 \mathrm{a}$ & $1.0 \pm 0.10 \mathrm{bc}$ & $0.8 \pm 0.06 \mathrm{c}$ \\
& Cone (total) & $2.5 \pm 0.21 \mathrm{bc}$ & $2.7 \pm 0.29 \mathrm{abc}$ & $3.2 \pm 0.21 \mathrm{ab}$ & $3.4 \pm 0.38 \mathrm{a}$ & $3.2 \pm 0.38 \mathrm{ab}$ & $2.9 \pm 0.15 \mathrm{abc}$ & $2.2 \pm 0.40 \mathrm{c}$ \\
\hline
\end{tabular}

Means followed by different letters in rows are significantly different by the Tukey HSD test $(\alpha=0.05)$

growing season. The lower average value $\left(2.1 \mathrm{~g} \mathrm{~kg}^{-1}\right)$ was recorded in plot 1 on June 2, and the higher average value $\left(15.3 \mathrm{~g} \mathrm{~kg}^{-1}\right)$ was found in plot 5 on September 1. Also, in spite of significant differences in leaf $\mathrm{Mg}$ concentration had been found in all sampling dates, a coherent trend among experimental plots with different plant vigour was not observed. As for $\mathrm{Ca}$, the levels of $\mathrm{Mg}$ in stems and cones were lower than those in the leaves taken at harvest.

The concentration of $\mathrm{Fe}$ in the leaves increased throughout the growing season (Table 6). On June 1, leaf Fe levels varied from $91.3 \mathrm{mg} \mathrm{kg}^{-1}$ (plot 1) to $153.4 \mathrm{mg} \mathrm{kg}^{-1}$ (plot 2) and, on September 1, from $169 \mathrm{mg} \mathrm{kg}^{-1}$ (plot 7) to $691.2 \mathrm{mg} \mathrm{kg}^{-1}$ (plot 4). In most sampling dates, significant differences were found in leaf $\mathrm{Fe}$ concentrations among the experimental plots. The plants with fair vigour in plot 4 present the highest $\mathrm{Fe}$ leaf concentration from July 1 until the harvest date in all leaf positions and in cones. The cones showed average values higher than those of the stems and lower than those of the leaves.

Leaf $\mathrm{Mn}$ concentrations ranged from a minimum of $36.1 \mathrm{mg} \mathrm{kg}^{-1}$ to a maximum of $542.3 \mathrm{mg} \mathrm{kg}^{-1}$ for plants of plots 3 and 1, respectively, both the records being from the harvest date (Table 7). Weak plants of plot 1 usually showed higher leaf
Mn values than the plants of good vigour of plot 7. Stems and cones presented lower average $\mathrm{Mn}$ values than the leaves.

The tissue concentrations in $\mathrm{Cu}, \mathrm{Zn}$ and $\mathrm{B}$ were also not presented in full tables. Leaf $\mathrm{Cu}$ concentrations significantly varied among experimental plots for most of the sampling dates. However, a clear relationship between leaf $\mathrm{Cu}$ levels and plant vigour was not observed. The lower $\left(2.1 \mathrm{mg} \mathrm{kg}^{-1}\right)$ and higher $\left(6.7 \mathrm{mg} \mathrm{kg}^{-1}\right)$ average values were found in the sampling of July 1 in plots 6 and 2, respectively. Stems and cones showed leaf $\mathrm{Cu}$ levels not much dissimilar than those of the leaves. However, cone $\mathrm{Cu}$ levels appeared generally higher than those of the other tissues.

In most of the sampling dates, leaf $\mathrm{Zn}$ concentrations significantly varied among the experimental plots. Plants of higher vigour (plots 6 and 7) usually showed lower leaf $\mathrm{Zn}$ levels. Between the plots of weak and fair plant vigour, no consistency in results was found, with the lower and higher values interchanging among sampling dates. Leaf $\mathrm{Zn}$ levels varied from $18.4 \mathrm{mg} \mathrm{kg}^{-1}$ (plot 6, July 1 sampling) to $448.2 \mathrm{mg} \mathrm{kg}^{-1}$ (plot 5, July 27 sampling). At harvest, leaf $\mathrm{Zn}$ levels tended to be lower than those of the previous sampling dates. Cones exhibited higher $\mathrm{Zn}$ levels than the leaves and stems.

Table 5 Tissue potassium concentrations $\left(\mathrm{g} \mathrm{kg}^{-1}\right)$ (mean \pm standard deviation) at different sampling dates and tissue position in the plant from the different experimental plots

\begin{tabular}{|c|c|c|c|c|c|c|c|c|}
\hline Date & $\begin{array}{l}\text { Tissue } \\
\text { (plant position) }\end{array}$ & Plot 1 (weak) & Plot 2 (weak) & Plot 3 (weak) & Plot 4 (fair) & Plot 5 (fair) & Plot 6 (good) & Plot 7 (good) \\
\hline June 2 & Leaf $(1 \mathrm{~m})$ & $13.9 \pm 1.01 \mathrm{ab}$ & $12.8 \pm 3.15 \mathrm{abc}$ & $7.9 \pm 1.35 \mathrm{c}$ & $15.8 \pm 2.46 \mathrm{ab}$ & $10.8 \pm 1.46 \mathrm{bc}$ & $10.7 \pm 1.25 \mathrm{bc}$ & $16.5 \pm 2.00 \mathrm{a}$ \\
\hline \multirow[t]{2}{*}{ Jul y1 } & Leaf $(1 \mathrm{~m})$ & $15.5 \pm 0.85 b$ & $10.1 \pm 1.21 \mathrm{~cd}$ & $6.7 \pm 1.02 \mathrm{e}$ & $13.3 \pm 1.08 \mathrm{bc}$ & $10.4 \pm 1.71 \mathrm{~cd}$ & $9.5 \pm 1.85 \mathrm{de}$ & $23.6 \pm 1.17 \mathrm{a}$ \\
\hline & Leaf $(2 \mathrm{~m})$ & $16.3 \pm 0.81 \mathrm{~b}$ & $10.8 \pm 0.45 \mathrm{~cd}$ & $7.5 \pm 1.15 \mathrm{~d}$ & $16.0 \pm 0.67 \mathrm{~b}$ & $13.2 \pm 2.03 b c$ & $10.5 \pm 2.16 \mathrm{~cd}$ & $29.2 \pm 1.99 \mathrm{a}$ \\
\hline \multirow[t]{2}{*}{ July 27} & Leaf $(1 \mathrm{~m})$ & $10.9 \pm 1.48 \mathrm{bc}$ & $7.1 \pm 0.49 \mathrm{~cd}$ & $5.0 \pm 0.44 \mathrm{~d}$ & $12.9 \pm 2.65 b$ & $7.0 \pm 3.87 \mathrm{~cd}$ & $7.6 \pm 2.10 \mathrm{bcd}$ & $22.0 \pm 2.06 \mathrm{a}$ \\
\hline & Leaf $(2 \mathrm{~m})$ & $15.1 \pm 0.25 \mathrm{~b}$ & $8.5 \pm 0.64 \mathrm{~cd}$ & $6.0 \pm 0.93 \mathrm{~d}$ & $16.3 \pm 1.27 \mathrm{bc}$ & $13.9 \pm 2.82 b c$ & $11.9 \pm 3.93 \mathrm{bcd}$ & $25.2 \pm 4.39 \mathrm{a}$ \\
\hline \multirow[t]{4}{*}{ September 1 (harvest) } & Leaf (basal) & $10.6 \pm 0.25 \mathrm{~b}$ & $5.1 \pm 0.56 \mathrm{~cd}$ & $3.6 \pm 0.20 \mathrm{~d}$ & $8.1 \pm 3.64 b c$ & $7.3 \pm 2.04 \mathrm{bcd}$ & $4.8 \pm 0.78 \mathrm{~cd}$ & $15.5 \pm 0.79 \mathrm{a}$ \\
\hline & Leaf (top) & $9.9 \pm 1.15 b$ & $5.4 \pm 0.36 \mathrm{~b}$ & $4.7 \pm 0.90 \mathrm{~b}$ & $8.1 \pm 3.55 b$ & $7.4 \pm 2.73 b$ & $6.9 \pm 2.76 b$ & $15.1 \pm 0.83 \mathrm{a}$ \\
\hline & Stem (total) & $11.4 \pm 1.70 \mathrm{a}$ & $4.2 \pm 0.64 \mathrm{bc}$ & $2.7 \pm 0.38 \mathrm{c}$ & $8.4 \pm 1.33 \mathrm{ab}$ & $8.3 \pm 3.30 \mathrm{ab}$ & $5.1 \pm 1.30 \mathrm{bc}$ & $10.1 \pm 2.05 \mathrm{a}$ \\
\hline & Cone (total) & $14.8 \pm 2.46 \mathrm{ab}$ & $10.9 \pm 0.56 b$ & $10.7 \pm 2.21 \mathrm{~b}$ & $14.1 \pm 1.06 \mathrm{ab}$ & $16.2 \pm 2.33 \mathrm{ab}$ & $13.1 \pm 2.49 \mathrm{ab}$ & $18.9 \pm 4.35 \mathrm{a}$ \\
\hline
\end{tabular}

Means followed by different letters in rows are significantly different by the Tukey HSD test $(\alpha=0.05)$ 
Table 6 Tissue iron concentrations $\left(\mathrm{mg} \mathrm{kg}^{-1}\right)$ (mean \pm standard deviation) at different sampling dates and tissue position in the plant from the different experimental plots

\begin{tabular}{|c|c|c|c|c|c|c|c|c|}
\hline Date & $\begin{array}{l}\text { Tissue } \\
\text { (plant } \\
\text { position) }\end{array}$ & Plot 1 (weak) & Plot 2 (weak) & Plot 3 (weak) & Plot 4 (fair) & Plot 5 (fair) & Plot 6 (good) & Plot 7 (good) \\
\hline June 2 & Leaf $(1 \mathrm{~m})$ & $91.3 \pm 4.6 \mathrm{a}$ & $153.4 \pm 120.9 \mathrm{a}$ & $144.9 \pm 55.7 \mathrm{a}$ & $126.4 \pm 4.5 \mathrm{a}$ & $93.4 \pm 4.0 \mathrm{a}$ & $93.7 \pm 15.9 \mathrm{a}$ & $118.8 \pm 30.4 \mathrm{a}$ \\
\hline \multirow[t]{2}{*}{ July 1} & Leaf $(1 \mathrm{~m})$ & $170.5 \pm 9.3 b$ & $171.8 \pm 21.1 \mathrm{~b}$ & $153.5 \pm 11.1 \mathrm{~b}$ & $264.0 \pm 28.8 \mathrm{a}$ & $148.1 \pm 22.9 \mathrm{~b}$ & $168.0 \pm 8.8 b$ & $156.6 \pm 15.2 \mathrm{~b}$ \\
\hline & Leaf (2 m) & $155.2 \pm 10.5 \mathrm{bc}$ & $134.6 \pm 11.5 \mathrm{bc}$ & $109.9 \pm 10.2 \mathrm{bc}$ & $304.8 \pm 75.2 \mathrm{a}$ & $198.2 \pm 38.9 b$ & $98.6 \pm 16.3 \mathrm{c}$ & $109.9 \pm 13.8 \mathrm{bc}$ \\
\hline \multirow[t]{2}{*}{ July 27} & Leaf $(1 \mathrm{~m})$ & $197.9 \pm 17.1 \mathrm{~b}$ & $187.2 \pm 14.5 \mathrm{bc}$ & $173.7 \pm 5.9 \mathrm{bc}$ & $266.4 \pm 17.8 \mathrm{a}$ & $180.5 \pm 8.2 \mathrm{bc}$ & $126.1 \pm 20.7 \mathrm{~d}$ & $155.8 \pm 14.1 \mathrm{~cd}$ \\
\hline & Leaf (2 m) & $174.6 \pm 17.7 b$ & $178.6 \pm 21.5 b$ & $141.1 \pm 4.9 \mathrm{bc}$ & $224.5 \pm 25.1 \mathrm{a}$ & $178.2 \pm 28.8 \mathrm{~b}$ & $106.8 \pm 1.0 \mathrm{c}$ & $122.8 \pm 5.9 \mathrm{c}$ \\
\hline \multirow{4}{*}{$\begin{array}{l}\text { September } \\
\quad 1 \\
\text { (harvest) }\end{array}$} & Leaf (basal) & $258.4 \pm 25.1 b$ & $256.1 \pm 12.9 \mathrm{~b}$ & $373.4 \pm 33.5 b$ & $691.2 \pm 136.1 \mathrm{a}$ & $313.5 \pm 24.0 \mathrm{~b}$ & $233.3 \pm 34.0 \mathrm{~b}$ & $294.7 \pm 12.1 \mathrm{~b}$ \\
\hline & Leaf (top) & $250.9 \pm 55.1 \mathrm{c}$ & $233.6 \pm 44.3 \mathrm{c}$ & $239.7 \pm 25.2 \mathrm{c}$ & $606.1 \pm 44.6 \mathrm{a}$ & $464.3 \pm 97.1 \mathrm{~b}$ & $237.8 \pm 51.1 \mathrm{c}$ & $169.4 \pm 7.4 \mathrm{c}$ \\
\hline & Stem (total) & $56.4 \pm 11.2 \mathrm{ab}$ & $65.0 \pm 9.9 \mathrm{ab}$ & $80.2 \pm 15.4 \mathrm{a}$ & $62.9 \pm 18.7 \mathrm{ab}$ & $40.8 \pm 4.5 b$ & $40.8 \pm 1.1 \mathrm{~b}$ & $67.2 \pm 1.1 \mathrm{ab}$ \\
\hline & Cone (total) & $180.1 \pm 49.9 \mathrm{a}$ & $151.2 \pm 17.2 \mathrm{a}$ & $183.7 \pm 26.9 \mathrm{a}$ & $315.0 \pm 30.2 \mathrm{a}$ & $242.9 \pm 178.1 \mathrm{a}$ & $239.5 \pm 36.8 \mathrm{a}$ & $138.8 \pm 21.4 \mathrm{a}$ \\
\hline
\end{tabular}

Means followed by different letters in rows are significantly different by the Tukey HSD test $(\alpha=0.05)$

Leaf B levels significantly varied among the experimental plots for all sampling dates. Plots 7, 6 and 5, but also plot 1, showed a dominance of ' $a$ ' letter from the Tukey test, meaning that the average values tended to be higher in these plots. Leaf B levels early in the growing season (June 2) were usually lower than those found in the samplings performed later. Considering all the sampling dates, leaf B levels varied from $20.1 \mathrm{mg} \mathrm{kg}^{-1}$ (plot 2, June 2 sampling) to $97.5 \mathrm{mg} \mathrm{kg}^{-1}$ (plot 7, September 1 sampling). Cones, and particularly stems, showed B levels lower than those found in the leaves.

\subsection{Principal Component Analysis}

\subsubsection{Soil PCA}

A PCA was applied to soil properties in order to assess the possible relationships between them and the experimental plots of different plant vigour. Exchangeable acidity and
$\mathrm{pH}_{\mathrm{KCl}}$ were not included in the analysis, since both presented very low eigenvalues and do not contribute to explained variance. Three components were retained from PCA that explained $80.3 \%$ of total variance. The first component (C1) explained $41.9 \%$ of the total variance, with a high Cronbach's alpha (0.91). The CEC had the highest positive loading in this component (1.1127), indicating high soil CEC values, and therefore, $\mathrm{C} 1$ was denominated as 'high CEC' (Table 8). The other variables associated with this dimension presented also positive and high loadings, with a predominance of exchangeable bases $(\mathrm{Ca}>\mathrm{Mg})$ and cationic micronutrients $(\mathrm{Zn}>\mathrm{Cu}>\mathrm{Fe})$, but also $\mathrm{pH}_{\mathrm{H}_{2} \mathrm{O}}$.

The second component (C2) explained $20.7 \%$ of total variance, with an acceptable Cronbach's alpha of 0.76 . The two variables with higher positive loadings in component $\mathrm{C} 2$ were extractable $\mathrm{K}_{\mathrm{ER}}$ and exchangeable $\mathrm{K}$, indicating soil with high $\mathrm{K}$ concentrations, and so $\mathrm{C} 2$ component was named 'high $\mathrm{K}$ '. $\mathrm{Na}, \mathrm{B}$ and extractable $\mathrm{P}_{\mathrm{ER}}$ also presented high positive loadings

Table 7 Tissue manganese concentrations $\left(\mathrm{m} \mathrm{kg}^{-1}\right)$ (mean \pm standard deviation) at different sampling dates and tissue position in the plant from the different experimental plots

\begin{tabular}{lllllllll}
\hline Date & $\begin{array}{l}\text { Tissue } \\
\text { (plant } \\
\text { position) }\end{array}$ & Plot 1 (weak) & Plot 2 (weak) & Plot 3 (weak) & Plot 4 (fair) & Plot 5 (fair) & Plot 6 (good) & Plot 7 (good) \\
& & & & & & & \\
& & & & & & \\
\hline June 2 & Leaf $(1 \mathrm{~m})$ & $320.2 \pm 33.2 \mathrm{a}$ & $253.4 \pm 50.6 \mathrm{ab}$ & $215.7 \pm 44.2 \mathrm{bc}$ & $143.5 \pm 5.9 \mathrm{c}$ & $176.1 \pm 11.3 \mathrm{bc}$ & $225.5 \pm 29.1 \mathrm{~b}$ & $68.5 \pm 12.6 \mathrm{~d}$ \\
July 1 & Leaf $(1 \mathrm{~m})$ & $296.0 \pm 33.7 \mathrm{a}$ & $288.0 \pm 45.7 \mathrm{a}$ & $115.0 \pm 12.1 \mathrm{bc}$ & $153.7 \pm 32.5 \mathrm{~b}$ & $165.9 \pm 15.6 \mathrm{~b}$ & $63.0 \pm 12.9 \mathrm{c}$ & $125.6 \pm 14.4 \mathrm{bc}$ \\
& Leaf $(2 \mathrm{~m})$ & $230.3 \pm 40.8 \mathrm{a}$ & $175.3 \pm 45.9 \mathrm{a}$ & $52.2 \pm 17.9 \mathrm{~b}$ & $50.6 \pm 14.9 \mathrm{~b}$ & $56.2 \pm 8.0 \mathrm{~b}$ & $45.7 \pm 6.7 \mathrm{~b}$ & $94.4 \pm 11.9 \mathrm{~b}$ \\
July 27 & Leaf $(1 \mathrm{~m})$ & $459.2 \pm 170.4 \mathrm{a}$ & $194.9 \pm 26.6 \mathrm{~b}$ & $63.4 \pm 7.5 \mathrm{~b}$ & $111.8 \pm 4.2 \mathrm{~b}$ & $143.8 \pm 25.8 \mathrm{~b}$ & $92.1 \pm 9.5 \mathrm{~b}$ & $181.0 \pm 11.5 \mathrm{~b}$ \\
& Leaf $(2 \mathrm{~m})$ & $420.7 \pm 18.3 \mathrm{a}$ & $129.5 \pm 27.5 \mathrm{bc}$ & $37.4 \pm 5.0 \mathrm{~d}$ & $46.9 \pm 7.1 \mathrm{~d}$ & $58.7 \pm 12.4 \mathrm{~d}$ & $95.8 \pm 7.6 \mathrm{c}$ & $158.1 \pm 13.5 \mathrm{~b}$ \\
September & Leaf (basal) & $536.5 \pm 130.3 \mathrm{a}$ & $113.1 \pm 1.7 \mathrm{~b}$ & $56.8 \pm 16.8 \mathrm{~b}$ & $136.2 \pm 25.0 \mathrm{~b}$ & $147.6 \pm 52.1 \mathrm{~b}$ & $131.6 \pm 20.7 \mathrm{~b}$ & $129.0 \pm 29.3 \mathrm{~b}$ \\
1 & Leaf (top) & $542.3 \pm 61.6 \mathrm{a}$ & $41.6 \pm 6.3 \mathrm{~b}$ & $36.1 \pm 13.8 \mathrm{~b}$ & $51.7 \pm 6.1 \mathrm{~b}$ & $86.4 \pm 42.2 \mathrm{~b}$ & $113.3 \pm 14.3 \mathrm{~b}$ & $81.8 \pm 38.8 \mathrm{~b}$ \\
(harvest) & Stem (total) & $257.1 \pm 89.7 \mathrm{a}$ & $39.8 \pm 6.1 \mathrm{~b}$ & $27.1 \pm 8.9 \mathrm{~b}$ & $24.9 \pm 5.7 \mathrm{~b}$ & $27.4 \pm 5.5 \mathrm{~b}$ & $37.7 \pm 5.8 \mathrm{~b}$ & $63.7 \pm 23.9 \mathrm{~b}$ \\
& Cone (total) & $175.6 \pm 37.1 \mathrm{a}$ & $47.0 \pm 3.6 \mathrm{~b}$ & $54.2 \pm 7.1 \mathrm{~b}$ & $54.8 \pm 3.2 \mathrm{~b}$ & $43.5 \pm 15.5 \mathrm{~b}$ & $84.2 \pm 18.6 \mathrm{~b}$ & $57.0 \pm 19.2 \mathrm{~b}$ \\
\hline
\end{tabular}

Means followed by different letters in rows are significantly different by the Tukey HSD test $(\alpha=0.05)$ 
Table 8 Components extracted with PCA applied to soil properties (C1, $\mathrm{C} 2$ and $\mathrm{C} 3$ ), and respective eigenvalues, explained variance, Cronbach's alpha values and component loadings of each soil parameter in each component

\begin{tabular}{llll}
\hline & \multicolumn{2}{l}{ Components } & \\
\cline { 2 - 4 } & C1-high CEC & C2-high K & C3-high Mn \\
\hline Eigenvalue & 7.120 & 3.514 & 3.011 \\
Explained variance (\%) & 41.9 & 20.7 & 17.7 \\
Cronbach's alpha & 0.913 & 0.760 & 0.710 \\
Component loadings & & & \\
Cation exchange capacity & 1.127 & -0.173 & -0.325 \\
Exchangeable Ca & 1.107 & -0.371 & -0.230 \\
Extractable $\mathrm{Zn}$ & 1.087 & -0.098 & 0.455 \\
Exchangeable $\mathrm{Mg}$ & 1.078 & -0.113 & -0.315 \\
Acid phosphatase & 1.033 & -0.050 & -0.482 \\
Extractable $\mathrm{Cu}$ & 0.948 & -0.745 & 0.047 \\
pH & 0.864 & -0.809 & 0.178 \\
Extractable $\mathrm{Fe}$ & 0.857 & -0.350 & 0.379 \\
Extractable $\mathrm{K}_{\mathrm{ER}}$ & -0.200 & 1.239 & -0.708 \\
Exchangeable $\mathrm{K}$ & 0.440 & 1.192 & -0.340 \\
Exchangeable $\mathrm{Na}$ & 0.687 & 0.992 & -0.241 \\
Extractable $\mathrm{B}$ & 0.592 & 0.885 & 0.672 \\
Extractable $\mathrm{P}_{\mathrm{ER}}$ & 0.696 & 0.874 & 0.613 \\
Extractable $\mathrm{Mn}$ & 0.200 & -0.152 & 1.412 \\
Conductivity & 0.367 & 0.008 & -1.085 \\
Organic C & 0.738 & -0.050 & -0.924 \\
Extractable $\mathrm{P}_{\mathrm{OL}}$ & 0.678 & 0.832 & 0.869 \\
\hline & & & \\
\hline
\end{tabular}

Values in italics correspond to the highest loading of each soil property in the respective component

ER Egner-Riehm method, $O L$ Olsen method

for this component. The third component (C3) explained $17.7 \%$ of total variance, also with an acceptable Cronbach's alpha of 0.71 . This component was named 'high Mn', because of strong and positive association of $\mathrm{Mn}$. Conductivity and organic C were strong and negatively associated with $\mathrm{C} 3$, indicating high soil $\mathrm{Mn}$ concentration associated with lower values for electrical conductivity and organic $\mathrm{C}$.

Considering that high scores in $\mathrm{C} 1$ indicate higher soil $\mathrm{CEC}$ values, high scores in $\mathrm{C} 2$ indicate higher soil $\mathrm{K}$ concentrations and high scores in $\mathrm{C} 3$ indicate higher soil Mn concentrations, and comparing the scores of each one of the plots in each one of the PCA components (Fig. 3), results suggest that

a) The plots with fair plant vigour presented the highest scores in $\mathrm{C} 1$ and thus the highest CEC values. The plots with good plant vigour presented the lowest CEC values, and the plots with weak plant vigour settle in the middle, with higher scores for plots 2 and 3 . In $\mathrm{C} 1$ component, the plots with the same vigour were more similar in between and dissimilar from the others; b) The plots with good (plot 7), fair (plots 5 and 4) and weak (plot 1) plant vigour presented the highest scores in $\mathrm{C} 2$ and thus the highest soil $\mathrm{K}$ concentrations, in decreasing order. C2 component did not explained variances between plant vigour, since plots with different plant vigour (weak, good and fair) did not differed statistically; and

c) The highest scores in C3, corresponding to the highest soil Mn concentration, were registered for plot 1 followed by plots 6 and 2. For $\mathrm{C} 3$, the similarity between plots with the same plant vigour only occurred for plant with fair vigour that registered middle scores.

\subsubsection{Plant PCA}

Principal component analysis was also performed from plant data and specifically for all sampling dates and analysed tissues. Three components were extracted in both PCA that explained a total variance of $70.3 \%$ for PCA in leaves data and $79.1 \%$ for PCA in harvest data. Table 9 provides an overview of both PCAs.

For PCA results in leaves collected until July 27, score means in each one of the PCA components and Tukey HSD test were calculated for all plots, regarding the three sampling dates and leaf positions (Fig. 4). The information was summarized as follows:

a) High scores in $\mathrm{C} 1$ component indicate, in the comparison between plots, lower leaf $\mathrm{P}$ concentrations associated with higher leaf concentrations of $\mathrm{Ca}, \mathrm{Mg}, \mathrm{B}$ and $\mathrm{Fe}$. The higher scores in $\mathrm{C} 1$ were recorded in the sample date of July 27 , and the lower on June 2, both for leaves collected at $1 \mathrm{~m}$. The plots with fair vigour (plots 5 and 4) generally presented higher scores in $\mathrm{C} 1$, and plots with weak vigour (plot 1) and with good vigour (plot 7) mostly presented the lower scores;

b) High scores in $\mathrm{C} 2$ component indicate higher leaf $\mathrm{Mn}$ concentrations, followed by higher $\mathrm{K}$ and $\mathrm{N}$ concentrations. Plot 1 (weak) registered the highest scores in all dates and leaf position, related with highest leaf Mn concentrations. Plot 7 (good) was also high on July 1 and 27, related with highest leaf $\mathrm{K}$ and $\mathrm{N}$ concentrations. All the other plots presented low scores; and

c) High scores in $\mathrm{C} 3$ component indicate higher $\mathrm{Cu}$ and $\mathrm{Zn}$ concentrations in leaves. In the first date (June 2), scores in $\mathrm{C} 3$ were positive and plot 3 (weak) presented the higher scores. Plot 7 mostly presented the lower scores, related with lower leaf $\mathrm{Cu}$ and $\mathrm{Zn}$ concentrations.

For PCA results in plants collected at harvest date, score means in each one of the PCA components and Tukey HSD 
Fig. 3 Distribution of scores and means in each one of the components obtained in PCA (C1, high cation exchange capacity; $\mathrm{C} 2$, high $\mathrm{K} ; \mathrm{C} 3$, high $\mathrm{Mn})$ for the soil collected in the plots (P) of weak (w), fair (f) and good (g) plant vigour. Means followed by different letters are statistically different by the Tukey HSD test $(\alpha<0.05)$
C1

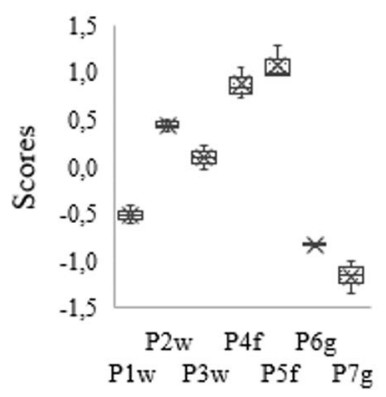

$\mathrm{C} 2$

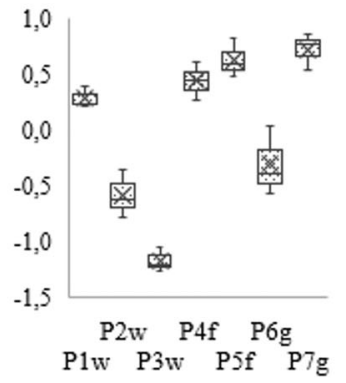

C3

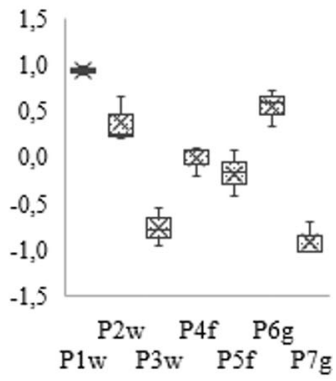

test were calculated for all plots, considering the different sample tissues (leaf basal, leaf top, stems and cones) (data not shown due to its extensity). The information may be summarized as follows:

a) High scores in $\mathrm{C} 1$ component indicate, in the comparison between plots, higher tissue $\mathrm{Ca}$ concentrations followed by tissue $\mathrm{B}, \mathrm{Mg}$ and Fe concentrations. The highest scores were registered in leaves and for plot 1 (weak), followed by plot 6 (good), explained mainly by leaf $\mathrm{Ca}$ and $\mathrm{B}$ concentrations. Scores were all negative for stems and cones;

b) High scores in $\mathrm{C} 2$ component indicate higher tissue $\mathrm{Zn}$ concentrations followed by $\mathrm{P}, \mathrm{N}$ and $\mathrm{Cu}$. The highest scores for $\mathrm{C} 2$ were registered for cones and for plots with fair vigour (plots 4 and 5) which differed significantly from plot 7 (good) with the lowest scores. These differences were related mainly with cone $\mathrm{Zn}, \mathrm{P}$ and $\mathrm{N}$ concentrations. The plots of weak vigour (plot 1) and fair vigour (plots 4 and 5) presented the highest scores in basal and top leaves, explained mostly by higher leaf $\mathrm{Zn}, \mathrm{P}$ and $\mathrm{Cu}$ concentrations and also $\mathrm{N}$ for plot 1 . Scores were negative for stems; and

c) High scores in C3 component indicate higher tissue Mn concentrations followed by higher K. For basal and top leaves, plot 1 (weak) presented the highest scores, followed by plot 7 (good), and both differed significantly from all the others. For cones and stems, plot 1 (weak) also presented the highest scores and differed significantly from all the others, followed by plot 7 (good). The higher scores for plot 1 were associated with high Mn concentrations and, for plot 7 , were associated with high $\mathrm{K}$ concentrations.

The three components indicated by each one of the PCAs performed in plant data were similar, but except for the component associated with high $\mathrm{Mn}$ and $\mathrm{K}$ concentrations, a common trend was not found. Mn and Fe seem to be the nutrients which differentiated more between plant vigour and also $\mathrm{K}$ at some extent.

\section{Discussion}

Soil analysis did not reveal particular problems for most of the soil properties analysed. Although low values sometimes appeared for exchangeable $\mathrm{Ca}, \mathrm{Mg}$ and $\mathrm{Na}$ and extractable $\mathrm{B}$, as compared with the Portuguese standard classification of soils (LQARS 2006), they were often associated with plots of plants with good vigour and not so much with plots of weak plants,

Table 9 PCA overview for the analysis performed in plant data with explained variance, Cronbach's alpha values and component summary

\begin{tabular}{|c|c|c|c|}
\hline & \multicolumn{3}{|l|}{ Components } \\
\hline & $\mathrm{C} 1$ & $\mathrm{C} 2$ & $\mathrm{C} 3$ \\
\hline \multicolumn{4}{|c|}{ PCA in leaves data from June 2 to July 27} \\
\hline Explained variance $(\%)$ & 38.4 & 16.7 & 15.2 \\
\hline Cronbach's alpha & 0.822 & 0.446 & 0.380 \\
\hline Component summary & Low $\mathrm{P}$ associated with high $\mathrm{Ca}, \mathrm{Mg}, \mathrm{B}$ and $\mathrm{Fe}$ & High $\mathrm{Mn}, \mathrm{K}$ and $\mathrm{N}$ & High $\mathrm{Cu}$ and $\mathrm{Zn}$ \\
\hline \multicolumn{4}{|l|}{ PCA in harvest data } \\
\hline Explained variance $(\%)$ & 36.7 & 30.5 & 11.9 \\
\hline Cronbach's alpha & 0.809 & 0.747 & 0.175 \\
\hline Component summary & High $\mathrm{Ca}, \mathrm{B}, \mathrm{Mg}$ and $\mathrm{Fe}$ & High $\mathrm{Zn}, \mathrm{P}, \mathrm{N}$ and $\mathrm{Cu}$ & High $\mathrm{Mn}$ and $\mathrm{K}$ \\
\hline
\end{tabular}


Fig. 4 Score means and standard deviations of the plots $(\mathrm{P})$ of weak (w), fair (f) and good (g) plant vigour in each one of the components obtained in PCA leaves data (C1 to $\mathrm{C} 3$ ) for the leaves collected at different tissue positions (leaf sampling dates of June 2, July 1 the same letter (leaf $1 \mathrm{~m}$ are in bold) are not statistically different $1 \mathrm{~m}$ and leaf $2 \mathrm{~m}$ ) and at the and July 27 . Means followed by

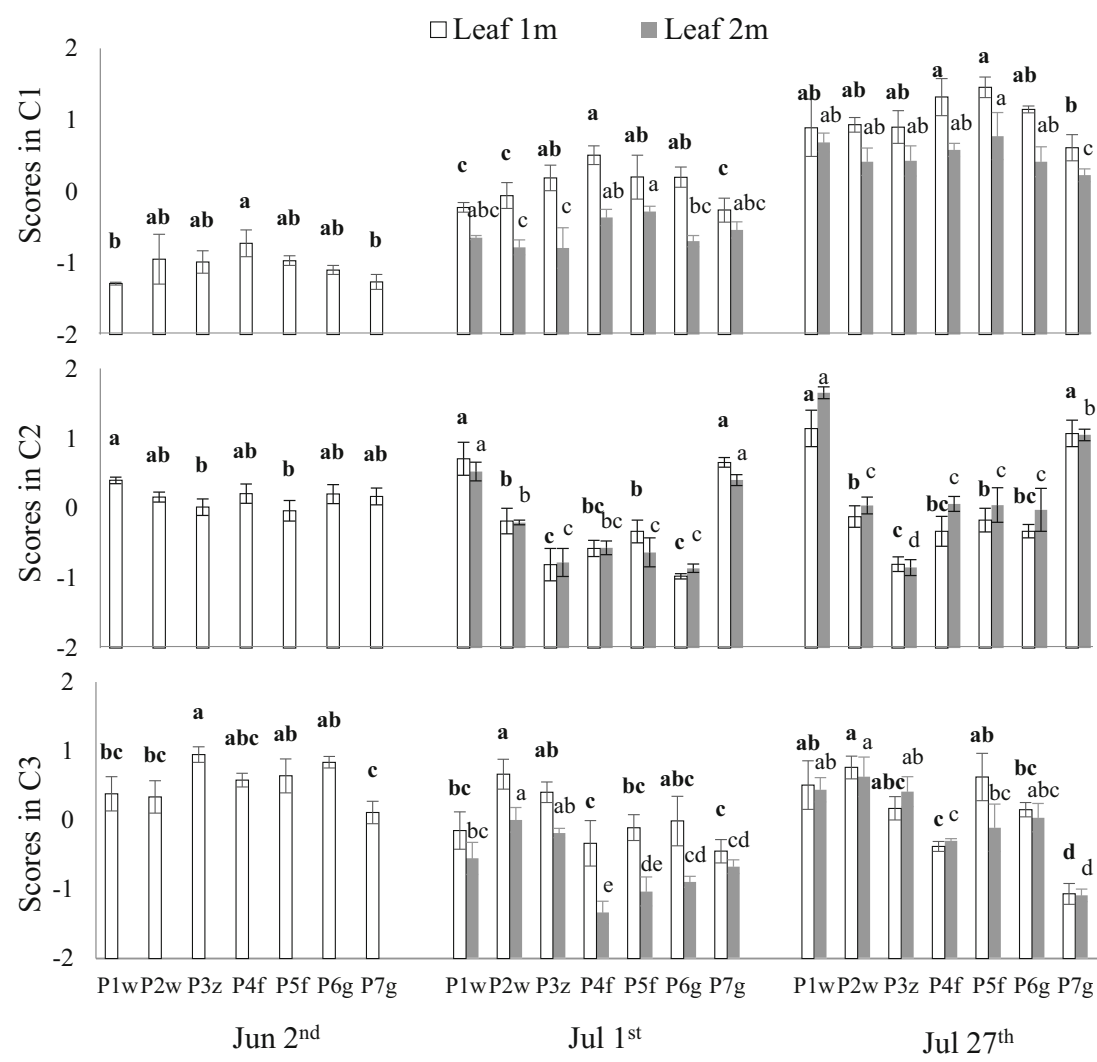

which reduces their meaning in relation to plant nutritional status. The extractable $\mathrm{P}$ and $\mathrm{K}$ were at relatively high levels in the soil and usually within the sufficiency ranges in the leaves, as reported by Bryson et al. (2014), which probably also excludes them from the list of soil properties that are negatively influencing plant growth, even though hop is considered to be a highly demanding $\mathrm{K}$ species (Gingrich et al. 1994). In turn, extractable $\mathrm{Mn}$ and Fe were at very high levels in the soils and appeared also at very high levels in plant tissues, frequently above the upper limit of the sufficiency range (Bryson et al. 2014), which gives them a great centrality in this study in the search for the causes affecting plant growth.

Excessive Mn levels were predominantly associated with plots of weak vigour plants and excessive Fe levels with plots of fair vigour plants. Reduction conditions may increase soluble forms of $\mathrm{Fe}$ and $\mathrm{Mn}$ in the soil due to the dissolution of $\mathrm{Fe}$ and Mn oxides, which can result in a strong uptake by plants (Phillips and Greenway 1998; Singer and Munns 2002). Stieger and Feller (1994) reported a considerable increase in $\mathrm{Mn}$ and $\mathrm{Fe}$ contents in wheat plants growing in large pots under conditions of flooding. Pot experiments are often associated with high levels of $\mathrm{Fe}$ and $\mathrm{Mn}$ in plant tissues (Afonso et al. 2018; Rodrigues et al. 2018), probably due to the greater regularity of water supply and poor drainage that occur with plants grown in the field.

Leaf Mn concentrations can vary greatly depending on the availability of $\mathrm{Mn}$ in the soil. In turn, the sufficiency ranges for
Mn or the critical Mn concentrations vary greatly among plant species (El-Jaoual and Cox 1998; Bryson et al. 2014). Symptoms of Mn toxicity can be observed at leaf Mn levels that range from $200 \mathrm{mg} \mathrm{kg}^{-1}$ in M- sensitive plants to over $5000 \mathrm{mg} \mathrm{kg}^{-1}$ in tolerant plants (Weil and Brady 2017). Hop is considered a sensitive species to high levels of $\mathrm{Mn}$, and a negative influence on plant growth has been reported mostly on acid soils (Gingrich et al. 1994).

Mn toxicity can manifest in plants by the reduction of biomass and early leaf senescence through biochemical disorders and, in particular, by damage of the photosynthetic system (Millaleo et al. 2010, 2019). In this study, leaf Mn concentrations above $200 \mathrm{mg} \mathrm{kg}^{-1}$ were often recorded, mostly on plots of weak plant vigour ( $>500 \mathrm{mg} \mathrm{kg}^{-1}$ at harvest in plot 1). The sufficiency ranges established for hop in vegetative and blossom stages, after Bryson et al. (2014), are $45-125 \mathrm{mg} \mathrm{kg}^{-1}$ and $50-150 \mathrm{mg} \mathrm{kg}^{-1}$, respectively. This is a strong evidence of the negative effect of $\mathrm{Mn}$ on crop growth and yield.

Excessive leaf $\mathrm{Fe}$ levels were also observed but particularly in plants of fair vigour. Maximum average values were recorded at $691.2 \mathrm{mg} \mathrm{kg}^{-1}$ in plot 4 from leaves sampled at harvest. The sufficiency range after Bryson et al. (2014) for leaf Fe in hop is between 35 and $151 \mathrm{mg} \mathrm{kg}^{-1}$. As far as we know, a description of a specific symptomology for Fe toxicity in hop does not exist, but the common symptom of Fe toxicity in crops is 'bronzing' (Broadley et al. 2012). The plots of fair vigour plants, those probably with toxic Fe levels in tissues, showed 
not only a reduction in total dry matter yield but also a notable reduction in cone yield, whose differences were not statistically significant in comparison to the plots of weak plants. Fe toxicity is a serious nutritional disorder in crop production on waterlogged soils; it is the second most severe yield-limiting factor in wetland rice (Broadley et al. 2012). Fe toxicity is usual in rice because of reduction processes associated with soil flooding (Sahrawat 2005). Fe toxicity in rice plants usually occurs to leaf Fe concentrations above $300 \mathrm{mg} \mathrm{kg}^{-1}$ (Fageria 2001). Plants with fair vigour in plots 5 and 4 registered leaf $\mathrm{Fe}$ concentrations at harvest higher than the values reported by Fageria (2001), which increases the evidence of the responsibility of $\mathrm{Fe}$ on the reduction in plant growth.

It seems that $\mathrm{Mn}$ will have been the more serious problem in the plots of weak plants and $\mathrm{Fe}$ in the plots of fair vigour plants. Antagonism between $\mathrm{Mn}$ and Fe has been widely reported (ElJaoual and Cox 1998; Fageria 2001; Rietra et al. 2017), and in spite of the physiological mechanisms not yet being clearly understood, it is currently accepted as occurring (Fageria 2001; Havlin et al. 2014). Also, an early report on hop from Thompson et al. (1950) showed that Fe deficiency was induced by high levels of Mn. This may mean that in weak vigour plants, the higher levels of $\mathrm{Mn}$ in plant tissue is the main reason for the reduced growth and for the relatively low Fe levels in tissue due to their antagonistic effect on Fe uptake. The lower levels of $\mathrm{Mn}$ in the plants of fair vigour plots may have allowed the increase in tissue Fe concentration, Fe being mainly responsible for the reduction of vigour in these plots.

One of the weaknesses of the surface irrigation methods is the heterogeneity in the spatial distribution of water (Walker 1989). Thus, winter flooding and the irrigation system seem to be the major causes for the appearance of patches of poorly developed plants and for the increased levels of $\mathrm{Mn}$ and $\mathrm{Fe}$ in soil and plants. Mn redox reactions are very sensitive to the potential redox of the soil (Sparrow and Uren 2014). In the thermodynamic sequence of the reduction process that takes place under flooding, Mn reduction occurs first, being followed by Fe reduction (Osman 2013). In most plots, leaf Mn levels were higher on the first sampling dates. In spite of irrigation, drier summer conditions seem to reduce Mn availability. This may mean that reduced soil drainage during winter has been more damaging to the development of plants than even the deficient water distribution associated with the surface irrigation system in summer. Leaf Fe levels tended to evolve in a different way with comparatively low values on the first sampling dates, probably due to antagonism by $\mathrm{Mn}$.

The risk of Mn toxicity increases in acidic soils, particularly under reduction conditions (Millaleo et al. 2010; Weil and Brady 2017). When soils are flooded, the $\mathrm{pH}$ of acidic soils tends to increase (Parent et al. 2008). However, the $\mathrm{pH}$ of the soils sampled on June 2 ranged from acidic $\left(\mathrm{pH}_{\mathrm{H}_{2} \mathrm{O}}=5.1\right)$ to slightly acidic $\left(\mathrm{pH}_{\mathrm{H}_{2} \mathrm{O}}=6.2\right)$, conditions that are still favourable for Mn toxicity to occur.
The presence of decomposable organic matter is one of the requirements for soil reduction (Pezeshki and DeLaune 2012). In submerged soils, the decomposition of organic matter is slower because it is overtaken mainly by anaerobic microorganisms, and large amounts of partially decomposed organic matter tend to accumulate (Weil and Brady 2017), influencing the degree of the reduction processes. Phillips and Greenway (1998) conducted an experiment under alternating waterlogged and drying conditions and concluded that in waterlogged soils, the magnitudes of changes, which included the increasing of soluble $\mathrm{Fe}, \mathrm{Mn}, \mathrm{Ca}$ and $\mathrm{Mg}$, were greatest in soils with the higher levels of organic matter. PCA indicated that high soil levels of Mn were strong and negatively associated with organic $\mathrm{C}$ and conductivity.

It is considered that $\mathrm{Mn}$ is closely related to the transformation of organic materials in soils and Mn availability under flooding is usually greater when soils contain high levels of organic matter (El-Jaoual and Cox 1998; Singer and Munns 2002). However, although $\mathrm{Mn}$ is released under reduction conditions, it is also removed by cation exchange reactions, precipitations and the formation of insoluble complexes (Millaleo et al. 2010). Ponnamperuma (1972) concluded that under reduction conditions when soils are high in $\mathrm{Mn}$ and low in organic matter, there can be high Mn peaks that will be late and broad; in contrast, in the case of high organic matter, the Mn peaks will be quicker and also quicker to decline. This seems to be the case in the present data: organic matter may play a role in the extension of high Mn concentrations during the growing season.

Conductivity was positively and strongly associated with organic $\mathrm{C}$ and negatively associated with $\mathrm{Mn}$. Soil electrical conductivity measures soil salinity in terms of the total concentration of the soluble salts (Lund et al. 1999). Usually, the electrical conductivity increases after flooding due to the increase in soluble $\mathrm{Fe}, \mathrm{Mn}$ and other cations (Camargo et al. 1999). The explanation for the negative correlation found with $\mathrm{Mn}$ might be related to Fe reduction. The first component extracted with PCA of the soil data indicated that higher values of CEC were associated with higher concentrations of exchangeable bases $(\mathrm{Ca}$ and $\mathrm{Mg})$ and cationic micronutrients ( $\mathrm{Zn}, \mathrm{Cu}$ and $\mathrm{Fe}$ ). Thus, the plots with higher Fe concentrations were also higher in CEC and other cationic micronutrients influencing conductivity values.

Soil texture varied among plots of the same plant vigour. Positive and strong correlations were found between CEC, exchangeable $\mathrm{Ca}$ and $\mathrm{Mg}$ with the clay content. PCA also indicated high CEC levels associated with high soil Fe concentrations. According to Singer and Munns (2002), in the majority of soils, the soluble Fe and Mn are mostly supplied by the dissolution of hydroxyoxides of the clay fraction. Favre et al. (2002), in a field experiment with rice, also observed a strong increase in CEC upon flooding. They attribute a CEC increase to changes in clay minerals as a result of octahedral 
$\mathrm{Fe}$ reduction and $\mathrm{Fe}$ oxyhydroxide coating solubilisation. They also concluded that CEC increases were reversible even in long-term flooding but may substantially modify soil chemical and even physical properties. Kostka et al. (1999) also suggested that structural $\mathrm{Fe}$ reduction is catalysed by soil anaerobic bacteria through the decrease of clay swelling and surface area and also through the increase of the clay surface charge density as a function of CEC. These findings are in agreement with the present data; the clay fraction seems to explain the predominance of $\mathrm{Fe}$ and CEC higher levels, particularly in the plots with fair plant vigour.

The availability of $\mathrm{P}$ and $\mathrm{K}$ in soil solution increases under reduction conditions (Phillips and Greenway 1998). In the present study, $\mathrm{K}$ and $\mathrm{P}$ concentrations in the soil were generally high but leaf concentrations were often below the sufficiency ranges, particularly for P. Stieger and Feller (1994) reported decreased levels of $\mathrm{K}$ and $\mathrm{P}$ in wheat shoots and grain under waterlogged conditions. In addition, the negative influence of high levels of $\mathrm{Mn}$ in soil solutions with $\mathrm{P}$ uptake has also been reported (El-Jaoual and Cox 1998).

The results also indicate variations in leaf $\mathrm{K}$ concentrations, with plot 7 (good) presenting the higher values and the plots of weak plant vigour generally the lower values, though in plot 6 (good), the leaf K levels were lower than in plot 1 (weak). This seems to be a contradictory result since $\mathrm{K}$ is usually associated with increasing plant growth (Hawkesford et al. 2012). However, $\mathrm{K}$ tends to vary greatly between cropping conditions and years (Rodrigues et al. 2020). Otherwise, probably this is due to the variability in soil reduction conditions along the fields and the subsequent nutrient interaction that takes place. Cationic antagonisms are predominant $(\mathrm{K}, \mathrm{Fe}, \mathrm{Mn}$, $\mathrm{Cu}$ and $\mathrm{Zn}$ ), but synergic interactions can also occur, as reported for Mn and K (Fageria 2001; Rietra et al. 2017). Plot 1 registered the higher $\mathrm{Mn}$ concentration in plant tissues, and among the plots of weak plant vigour, it also showed the higher tissue $\mathrm{K}$ concentration, suggesting the occurrence of a synergetic effect between $\mathrm{K}$ and $\mathrm{Mn}$, which is supported by PCA results.

Nutrient interactions are complex. They can influence plant growth by inducing deficiencies or toxicities, and they are particularly relevant under reduction conditions (Fageria 2001; Rietra et al. 2017). Mn toxicity, for instance, is highly dependent on environmental conditions and plant nutritional status and can be lowered by the increase of $\mathrm{Fe}, \mathrm{Ca}, \mathrm{Mg}, \mathrm{P}$ and K (El-Jaoual and Cox 1998; Auda et al. 2002). Mn enters the root cells through a specific transporter protein with little competition by other cations, although high concentrations of $\mathrm{Ca}$ and $\mathrm{Mg}$ adsorbed to the apoplastic cell wall of the root, particularly at high $\mathrm{pH}$, can reduce $\mathrm{Mn}$ adsorption and transport (Havlin et al. 2014). The results of PCA for soil data indicated $\mathrm{pH}, \mathrm{Ca}, \mathrm{Mg}$ and $\mathrm{Fe}$ with the higher and positive scores in the same component (C1-high CEC) and with low or negative scores in the other components (C2-kigh $\mathrm{K}$; C2-high $\mathrm{Mn}$ ).
These associations were also exposed in PCA results for plant data, underlining $\mathrm{Fe}, \mathrm{Ca}$ and $\mathrm{Mg}$ antagonism in relation with $\mathrm{Mn}$ and $\mathrm{K}$.

There are several factors that affect plant grown under stress flooding which cannot probably be fully elucidated through soil and plant analysis. When plant roots are submerged under water for a short term, the $\mathrm{O}_{2}$ levels decrease below optimal levels (hypoxia), but under long-term flooding, a complete lack of $\mathrm{O}_{2}$ can occur (anoxia) (Sairam et al. 2008). The $\mathrm{O}_{2}$ deprivation that takes place readily after soil flooding along with the increase of $\mathrm{CO}_{2}$ and the decrease of the redox potential can reduce root elongation and penetration depth (Irfan et al. 2010; Pezeshki and DeLaune 2012). Flooding per se changes also the physical properties of the soil which may increase the mechanical resistance to root penetration (Blom and Voesenek 1996). As a result of reduction conditions, root dysfunction, death and blockages due to phytotoxin damage can lead to inhibition of nutrient uptake and transport, nutrient toxicity and reduced water uptake (Pezeshki and DeLaune 2012). Mn and Fe seem to accumulate in roots in flooded soils, although $\mathrm{Mn}$ is less effectively retained (Millaleo et al. 2010; Alhdad et al. 2015). It has been reported that $\mathrm{Mn}$ toxicity can result in the shortening of the root and shoot length, with particular severity in the former (El-Jaoual and Cox 1998; Auda et al. 2002). In the conditions of these fields, it seems clear that some patches experienced anoxia during winter months.

\section{Conclusions}

The results indicate high soil and tissue Mn levels associated with the plots of weak vigour plants and high Fe levels associated with fair vigour plants. The toxic levels of $\mathrm{Mn}$ and $\mathrm{Fe}$ seem to result from soil reduction conditions due to the poor drainage of the soils and deficient water distribution caused by the surface irrigation system. Thus, the poor development of the plants is probably the result of the effects of flooding, giving rise to oxygen deprivation in the rooting zone and the consequent toxicity of $\mathrm{Mn}$ and Fe. Several factors, in particular the redox potential, $\mathrm{pH}$, clay content, organic $\mathrm{C}$ and nutrient interactions, seem to play a role in the prevalence of the effects of Mn over $\mathrm{Fe}$, and vice versa in different plots. In the light of the results, farmers should consider implementing a drainage system to improve soil aeration, to change the irrigation system to introduce drip irrigation and to increase soil $\mathrm{pH}$ by liming. Taken together, these factors can reduce the bioavailability of $\mathrm{Mn}$ and $\mathrm{Fe}$ and favour plant growth. If these changes are made, organic matter could also be favourably applied.

Acknowledgements The authors are grateful for the kindness and the logistical conditions that Mr. António Rodrigues and Mr. Sá Morais made available to the research team to carry out this work on their farms. 
Availability of Data and Material The datasets generated during and/or analysed during the current study are available from the corresponding author on reasonable request.

Authors' Contributions All authors contributed to the study conception and design. Material preparation and data collection were performed by Sandra Afonso, M. Ângelo Rodrigues and Margarida Arrobas, and analysis was performed by Sandra Afonso. The first draft of the manuscript was written by Sandra Afonso, and all authors commented on previous versions of the manuscript. All authors read and approved the final manuscript.

Funding Information The authors are grateful to the Foundation for Science and Technology (FCT, Portugal) and European Regional Development Fund (ERDF) under Programme PT2020 for the financial support to CIMO (UIDB/00690/2020) and also the scholarship grant of Sandra Afonso (number BD/116593/2016) under the Programme PT2020 (UID/AGR/00690/2019).

\section{Compliance with Ethical Standards}

Conflict of Interest The authors declare that they have no conflict of interest.

\section{References}

Afonso S, Arrobas M, Ferreira IQ, Rodrigues MÂ (2018) Leaf nutrient concentration standards for lemon verbena (Aloysia citrodora Paláu) obtained from field and pot fertilization experiments. J Appl Res Med Aromat Plants 8:33-40. https://doi.org/10.1016/j.jarmap. 2017.09.004

Alhdad GM, Zörb C, Al-Azzawi MJ, Flowers TJ (2015) Is the reduced growth of the halophyte Suaeda maritima under hypoxia due to toxicity of iron or manganese? Environ Exp Bot 116:61-70. https://doi.org/10.1016/j.envexpbot.2015.03.002

Auda MMA, Symeonidis L, Hatzistavrou E, Yupsanis T (2002) Nucleolytic activities and appearance of a new DNase in relation to nickel and manganese accumulation in Alyssum murale. J Plant Physiol 159:1087-1095. https://doi.org/10.1078/0176-1617-00667

Bindraban PS, Dimkpa C, Nagarajan L, Roy A, Rabbinge R (2015) Revisiting fertilisers and fertilisation strategies for improved nutrient uptake by plants. Biol Fertil Soils 51:897-911. https://doi.org/10. 1007/s00374-015-1039-7

Blom CW, Voesenek LA (1996) Flooding: the survival strategies of plants. Trends Ecol Evol 11:290-295. https://doi.org/10.1016/ 0169-5347(96)10034-3

Broadley M, Brown P, Cakmak I, Rengel Z, Zhao F (2012) Function of nutrients: micronutrients. In: Marschner P (ed) Marschner's mineral nutrition of higher plants. Elsevier, Amsterdam, pp 191-248

Bryson G, Mills H, Sasseville D, Jones JB Jr, Barker A (2014) Plant analysis handbook III: a guide to sampling, preparation, analysis and interpretation for agronomic and horticultural crops vol. VIII. Micro-Macro, Georgia

Camargo FAO, Santos GA, Zonta E (1999) Alterações eletroquímicas em solos inundados. J Ciência Rural 29:171-180. https://doi.org/10. 1590/S0103-84781999000100032

Čeh B, Čremožnik B (2015) Soil pH and hop (Humulus lupulus) yield related to liming material rate. Hmeljarski Bilten 22:49-57

Clescerl L, Greenberg A, Eaton A (1998) Standard methods for water and wastewater analysis. American Public Health Association

Eivazi F, Tabatabai M (1977) Phosphatases in soils. Soil Biol Biochem 9: $167-172$
El-Jaoual T, Cox DA (1998) Manganese toxicity in plants. J Plant Nutr 21:353-386. https://doi.org/10.1080/01904169809365409

Fageria VD (2001) Nutrient interactions in crop plants. J Plant Nutr 24: 1269-1290. https://doi.org/10.1081/Pln-100106981

Favre F, Tessier D, Abdelmoula M, Genin JM, Gates WP, Boivin P (2002) Iron reduction and changes in cation exchange capacity in intermittently waterlogged soil. Eur J Soil Sci 53:175-183. https:// doi.org/10.1046/j.1365-2389.2002.00423.x

Gao L, Su J, Tian Q, Shen Y (2020) Contrasting strategies of nitrogen absorption and utilization in alfalfa plants under different water stress. J Soil Sci Plant Nutr. https://doi.org/10.1007/s42729-02000230-0

George E, Horst WJ, Neumann E (2012) Adaptation of plants to adverse chemical soil conditions. In: Marschner P (ed) Marschner's mineral nutrition of higher plants. Elsevier, Amsterdam, pp 409-472

Gingrich C, Hart J, Christensen N (1994) Hops fertilizer guide. In: OSU extension catalog. Oregon State University, Extension Service. Available at https://catalog.extension.oregonstate.edu/fg79/html. ()

Havlin JL, Tisdale SL, Nelson WL, Beaton JD (2014) Soil fertility and fertilizers, an introduction to nutrient managemnt, 8th edn. Pearson, Boston

Hawkesford M, Horst W, Kichey T, Lambers H, Schjoerring J, Møller IS, White P (2012) Functions of macronutrients. In: Marschner P (ed) Marschner's mineral nutrition of higher plants. Elsevier, Amsterdam, pp 135-189

IPMA (2020) Normais climatológicas. Instituto Português do Mar e da Atmosfera. Accessed on April 2020. http://www.ipma.pt/pt/oclima/ normais.clima/

Irfan M, Hayat S, Hayat Q, Afroz S, Ahmad A (2010) Physiological and biochemical changes in plants under waterlogging. Protoplasma 241:3-17. https://doi.org/10.1007/s00709-009-0098-8

Kostka JE, Wu J, Nealson KH, Stucki JW (1999) The impact of structural $\mathrm{Fe}$ (III) reduction by bacteria on the surface chemistry of smectite clay minerals. Geochim Cosmochim Ac 63:3705-3713. https://doi. org/10.1016/S0016-7037(99)00199-4

Lakanen E, Erviö R (1971) A comparison of eight extractants for the determination of plant available micronutrients in soils. Helsingin yliopiston rehtorin professori Erkki Kivisen juhlajulkaisu/Viljo Puustjärvi

Lipecki J, Berbeć S (1997) Soil management in perennial crops: orchards and hop gardens. Soil Tillage Res 43:169-184

LQARS (2006) Manual de fertilização das culturas. In: Instituto Nacional de Investigação Agrária e das Pescas-Laboratório Químico Agrícola Rebelo da Silva

Lund E, Christy C, Drummond P (1999) Practical applications of soil electrical conductivity mapping. Precis Agric 99:771-779

Millaleo R, Reyes-Diaz M, Ivanov AG, Mora ML, Alberdi M (2010) Manganese as essential and toxic element for plants: transport, accumulation and resistance mechanisms. J Soil Sci Plant Nutr 10: 476-494. https://doi.org/10.4067/S0718-95162010000200008

Millaleo R et al (2019) Mn toxicity differentially affects physiological and biochemical features in highbush blueberry (Vaccinium corymbosum L.) cultivars. J Soil Sci Plant Nutr. https://doi.org/10. 1007/s42729-019-00166-0

Osman KT (2013) Soils: principles, properties and management. Springer Science \& Business Media https://doi.org/10.1007/978-94-007$5663-2$

Parent C, Capelli N, Berger A, Crèvecoeur M, Dat JF (2008) An overview of plant responses to soil waterlogging. Plant Stress 2:20-27

Pezeshki SR, DeLaune RD (2012) Soil oxidation-reduction in wetlands and its impact on plant functioning. Biology 1:196-221. https://doi. org/10.3390/biology 1020196

Phillips I, Greenway M (1998) Changes in water-soluble and exchangeable ions, cation exchange capacity, and phosphorusmax in soils under alternating waterlogged and drying conditions. Commun 
Soil Sci Plant Anal 29:51-65. https://doi.org/10.1080/ 00103629809369928

Ponnamperuma FN (1972) The chemistry of submerged soils. In: Advances in agronomy, vol 24. Elsevier, pp 29-96

Rietra RPJJ, Heinen M, Dimkpa CO, Bindraban PS (2017) Effects of nutrient antagonism and synergism on yield and fertilizer use efficiency. Commun Soil Sci Plant Anal 48:1895-1920. https://doi.org/ 10.1080/00103624.2017.1407429

Rodrigues M, Morais J (2015) A cultura do lúpulo em Bragança. Aspetos agronómicos inovadores e potencial e expansão. Livro de atas das Jornadas do Lúpulo e da Cerveja: novas oportunidades de negócio, pp 63-70

Rodrigues MÂ, Ferreira IQ, Afonso S, Arrobas M (2018) Sufficiency ranges for lemon balm and nutrient removals in aboveground phytomass. J Plant Nutr 41:996-1008. https://doi.org/10.1080/ 01904167.2018.1431671

Rodrigues MA, Grade V, Barroso V, Pereira A, Cassol LC, Arrobas M (2020) Chestnut response to organo-mineral and controlled-release fertilizers in rainfed growing conditions. J Soil Sci Plant Nutr 20: 380-391. https://doi.org/10.1007/s42729-019-00119-7

Rodrigues M, Morais J, Castro JP (2016) Growing hops in Bragança (NE Portugal): past, present and future. In: International conference on research for sustainable development in mountain regions. Instituto Politécnico de Bragança, pp 188-188

Sahrawat K (2005) Iron toxicity in wetland rice and the role of other nutrients. J Plant Nutr 27:1471-1504. https://doi.org/10.1081/ PLN-200025869

Sairam RK, Kumutha D, Ezhilmathi K, Deshmukh PS, Srivastava GC (2008) Physiology and biochemistry of waterlogging tolerance in plants. Biol Plant 52(3):401-412. https://doi.org/10.1007/s10535008-0084-6

Samson ME, Menasseri-Aubry S, Chantigny MH, Angers DA, Royer I, Vanasse A (2019) Crop response to soil management practices is driven by interactions among practices, crop species and soil type.
Field Crop Res 243:107623. https://doi.org/10.1016/j.fcr.2019. 107623

Singer MJ, Munns DN (2002) Soils: an introduction, 5th edn. Prentice Hall, Upper Saddle River

Sparrow LA, Uren NC (2014) Manganese oxidation and reduction in soils: effects of temperature, water potential, $\mathrm{pH}$ and their interactions. Soil Res 52:483-494. https://doi.org/10.1071/Sr13159

Stieger PA, Feller U (1994) Nutrient accumulation and translocation in maturing wheat plants grown on waterlogged soil. Plant Soil 160: 87-95. https://doi.org/10.1007/Bf00150349

Tabatabai M, Bremner J (1969) Use of p-nitrophenyl phosphate for assay of soil phosphatase activity. Soil Biol Biochem 1:301-307

Tan KH (2011) Principles of soil chemistry, 4th edn. CRC, New York

Thompson F, Cripps E, Burgess A (1950) The effect of soil acidity on growth of hops. Some observations on manganese toxicity and induced iron deficiency. Department of Hop Research, Wey College, pp 43-47

Van Reeuwijk L (2002) Procedures for soil analysis. Technical reports 9. International Soil Reference and Information

Walinga I, Van Vark W, Houba V, Van der Lee J (1989) Soil and plant analysis. Part 7: plant analysis procedures. Wageningen Agricultural University, Wageningen

Walker WR (1989) Guidelines for designing and evaluating surface irrigation systems. FAO irrigation and drainage paper 45 . Rome, Italy

Weil RR, Brady NC (2017) The nature and properties of soils, 15th edn. Global Edition, London

Xia L, Li X, Ma Q, et al. (2019) Simultaneous quantification of $\mathrm{N}_{2}, \mathrm{NH}_{3}$ and $\mathrm{N}_{2} \mathrm{O}$ emissions from a flooded paddy field under different $\mathrm{N}$ fertilization regimes. Glob Chang Biol https://doi.org/10.1111/gcb. $14958,26,2292,2303$

Publisher's note Springer Nature remains neutral with regard to jurisdictional claims in published maps and institutional affiliations. 